\title{
A Model of Pavlovian Eyelid Conditioning Based on the Synaptic Organization of the Cerebellum
}

\author{
Michael D. Mauk ${ }^{1,3}$ and Nelson H. Donegan ${ }^{2}$ \\ ${ }^{1}$ Department of Neurobiology and Anatomy \\ The University of Texas Medical School at Houston \\ Houston, Texas 77030 \\ ${ }^{2}$ Department of Psychology \\ Yale University \\ New Haven, Connecticut 06520
}

\section{Abstract}

We present a model based on the synaptic and cellular organization of the cerebellum to derive a diverse range of phenomena observed in Pavlovian eyelid conditioning. These phenomena are addressed in terms of critical pathways and network properties, as well as the sites and rules for synaptic plasticity. The theory is based on four primary hypotheses: (1) Two cerebellar sites of plasticity are involved in conditioning: (a) bidirectional long-term depression/potentiation at granule cell synapses onto Purkinje cells $(\mathrm{gr} \rightarrow \boldsymbol{P} \boldsymbol{k j})$ in the cerebellar cortex and (b) bidirectional plasticity in the interpositus nucleus that is controlled by inhibitory inputs from Purkinje cells; (2) climbing fiber activity is regulated to an equilibrium level at which the net strength of $\boldsymbol{g r} \rightarrow P k j$ synapses remains constant unless an unexpected unconditioned stimulus (US) is presented or an expected US is omitted; (3) a time-varying representation of the conditioned stimulus (CS) in the cerebellar cortex permits the temporal discrimination required for conditioned response timing; and (4) the ability of a particular segment of the CS to be represented consistently across trials varies as a function of time since $\mathrm{CS}$ onset. This variation in across-trials consistency is thought to contribute to the ISI function. The model suggests several

${ }^{3}$ Corresponding author. empirically testable predictions, some of which have been tested recently.

An initial challenge in understanding the neural mechanisms of associative learning phenomena is to identify the brain structures and pathways that are responsible for the acquisition and expression of a learned behavior. This difficult task generally involves a reductionistic strategy in which lesions, electrical stimulation, neural recordings, and neuroanatomical tracing techniques are used to identify neural structures and connections responsible for learning. A subsequent challenge is to identify the ways in which neural components interact to produce the range of observed learning phenomena. This requires an integrative approach in which critical features of the system are described with sufficient accuracy and detail to derive the target behaviors. One approach to this task is the use of hypothetical models that attempt to derive accounts for behavioral phenomena by combining features of the known synaptic organization and physiology of the brain regions thought to be involved with explicitly stated assumptions regarding how those regions are engaged by training. Such models can be useful, even before localization issues are completely resolved, by highlighting structural and functional properties that could produce learning, by generating testable predictions, and by pointing to the kinds of experiments that would be especially important for further progress.

Here, we outline a cerebellar model of Pavlovian eyelid conditioning based on the seminal cerebellar theories of Marr (1969) and Albus (1971). Our focus on the cerebellum and related brainstem structures is motivated by the diverse range of experiments suggesting that the cerebellum is

LEARNING \& MEMORY 3:130-158 @ 1997 by Cold Spring Harbor Laboratory Press ISSN1072-0502/97 \$5.00

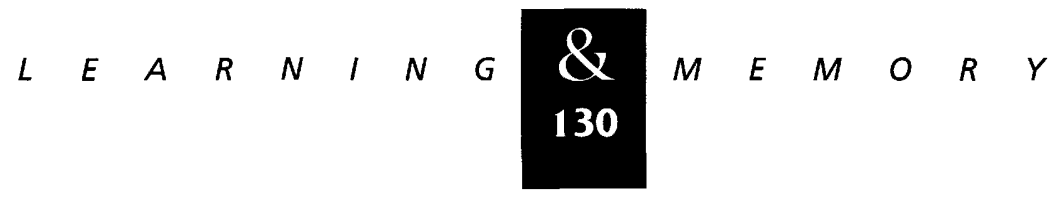


an element in the pathway that is activated by the conditioned stimulus (CS) and that drives the expression of conditioned responses (CRs) (the $\mathrm{CS} \rightarrow$ CR pathway). As outlined below, numerous studies suggest that the $\mathrm{CS} \rightarrow \mathrm{CR}$ pathway involves the particular pontine nuclei neurons activated by the CS, their mossy fiber projections to the cerebellum, the cerebellar projections to red nucleus, and the relevant motor nuclei (see Fig. 2, below). Other studies indicate that the unconditioned stimulus (US) activates climbing fiber inputs to the cerebellum. These observations have been interpreted as evidence that with appropriately timed mossy fiber and climbing fiber activation by the CS and US, respectively, the CS acquires the ability to activate the cerebellar interpositus nucleus neurons responsible for eliciting eyelid closure.

The primary support for these assumptions are (1) The retention of CRs is spared by decerebration (Mauk and Thompson 1987); (2) stimulation of appropriate regions of the anterior interpositus nucleus in trained or untrained animals elicits robust eyelid responses at modest stimulation parameters (McCormick and Thompson 1984); (3) lesions of the anterior interpositus nucleus abolish the expression of CRs (e.g., McCormick et al. 1982; McCormick and Thompson 1984; Yeo et al. 1985a; Welsh and Harvey 1989, 1992); (4) lesions of climbing fibers produce effects that mimic the absence of the US (McCormick et al. 1985; although, see Yeo et al. 1986); (5) lesions of mossy fibers can mimic removal of the CS (Steinmetz et al. 1987, 1988); (6) stimulation of mossy fibers and climbing fibers can substitute for the CS and US, respectively, to promote acquisition of CRs that are abolished by subsequent lesions of the anterior interpositus (Mauk et al. 1986; Steinmetz et al. 1989; Steinmentz 1990); and (7) reversible inactivation of the anterior interpositus nucleus retards learning (Krupa et al. 1993; Nordholm et al. 1993).

Thus, the view that the cerebellum is a necessary element of the CS $\rightarrow$ CR pathway is supported by evidence that information about the CS and US is conveyed to the cerebellum by separate afferent systems, that disrupting cerebellar output abolishes CRs, and that cerebellar output is capable of driving the expression of responses. Moreover, the convergence of CS and US information at the cerebellum combined with the ability of cerebellar output to elicit the expression of eyelid responses makes the cerebellum a strong candidate for the sites of plasticity that are responsible for the acqui- sition and expression of conditioned eyelid responses. A natural extension of this view is that certain behavioral properties of eyelid conditioning reflect the input/output processing properties of the cerebellum.

However, recent studies have challenged this cerebellar hypothesis and motivated the counterhypothesis that the cerebellum projects to, but is not a part of, a CS $\rightarrow$ CR pathway in the brain stem (Welsh and Harvey 1989, 1991; Kelly et al. 1990; Llinás and Welsh 1993; see also Bower and Kassel 1990). The influence that the cerebellum may have on these pathways is hypothesized to be modulatory, affecting either the performance of the responses (Welsh and Harvey 1991) or sensory processing (Gao et al. 1996). In this view, lesions of the cerebellum are thought to abolish CRs by disrupting the normal functioning of the critical brainstem pathways or of critical sensory discrimination (but, for other views, see Lavond et al. 1993 or Thompson and Krupa 1994).

Thus, whereas even the basic issue of cerebellar involvement in eyelid conditioning is not resolved to everyone's satisfaction, we suggest that another useful approach is to explore the feasibility of the mechanisms associated with each hypothesis. As a complement to additional empirical experiments evaluating whether the cerebellum (or brain stem) is responsible for eyelid conditioning, we can also ask whether the synaptic organization and physiology of the cerebellum (or brain stem) comprise a system with the capacity to generate the corpus of experimental data. To the extent that these questions yield awkward, biologically implausible answers, the hypothesis may be flawed. On the other hand, showing that the proposed cerebellar (or brain stem) mechanisms could generate the behavior in a biologically plausible manner would demonstrate that the system is, in principle, capable of explaining the facts of conditioning.

The development of the present model was driven by two well-characterized data sets: the rich literature describing in detail the synaptic organization of the cerebellum (e.g., Eccles et al. 1967; Ito 1984) and the extensively documented behavioral properties of eyelid conditioning (e.g., Wagner 1969; Gormezano 1972; Moore 1972; Siegel 1972; Gormezano et al. 1983, 1987; Kehoe and Napier 1991a,b; Napier et al. 1992; Kehoe et al. 1993a,b). Although we have incorporated known aspects of cerebellar anatomy and physiology into this model and have attempted to make as few

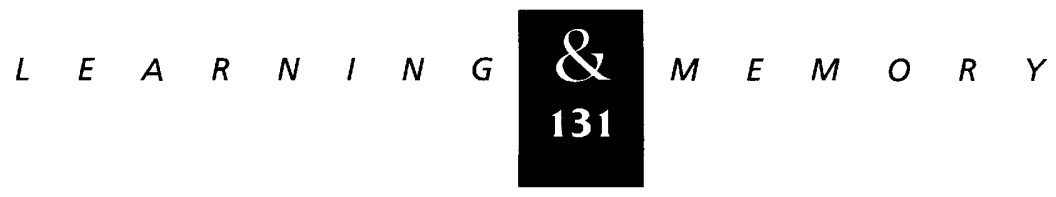


gratuitous assumptions as possible, we emphasize the hypothetical nature of this work. Our goal is to examine the degree to which biologically reasonable accounts of conditioning can be obtained from known principles of cerebellar neural processing.

\section{Basic Properties of Eyelid Conditioning}

Acquisition of conditioned eyelid responses requires the paired presentation of the CS and US. Typically, a relatively neutral stimulus such as a tone serves as the CS, and a periorbital shock or an air puff directed at the eye serves as the US (Fig. 1A). Initially, the US elicits an eyelid response, whereas there is no measurable eyelid response to the CS. With repeated CS + US pairings, the CS acquires the ability to elicit conditioned eyelid responses (Fig. 1B,C) that are timed to peak near US onset (Fig. 1E). The rate of acquisition and the timing of the CRs are influenced strongly by the interstimulus interval (ISI) between CS and US onsets (Schneiderman and Gormezano 1964; Schneiderman 1966; Frey and Ross 1968; Smith 1968; Salafia et al. 1979). The CS onset must precede the US by at least $80 \mathrm{msec}$ (i.e., ISI $>80 \mathrm{msec}$ ) but not by $>2-3 \mathrm{sec}$ for response to develop (Fig. 1D).

\section{Core Assumptions and Basic Architecture of the Model}

A basic organizing principle of the model is the hypothesis that the CS activates mossy fibers, the US activates climbing fibers, and output from the interpositus nucleus activates neurons that generate the conditioned eyelid responses (Fig. 2). In terms of the CS representation conveyed to the cerebellum, we assume that (1) each CS is encoded by activity in a subset of mossy fibers; (2) these subsets share common elements (active mossy fi-

\footnotetext{
${ }^{1}$ Recordings from mossy fibers activated by auditory stimuli have demonstrated a limited amount of temporal variation during presentation of tones (Aitkin and Boyd 1978). In general, these neurons respond phasically at tone onset and offset, tonically, or phasic/tonic. These variations over time are not in a range that can directly account for the range CR timing supported by various ISIs
}

bers) to the extent the CSs are similar; and (3) the mossy fiber CS representation conveys minimal temporal information, no more than might be provided by three categories of mossy fiber inputs, those that show either tonic, phasic, or phasic/ tonic responses to the $\mathrm{CS} .{ }^{1}$ The reinforcing properties of the US are assumed to be generated by the activation of climbing fibers that are specific for the US; for example, air puff to the eye activates climbing fibers from the facial regions of the inferior olive (IO) (Mauk et al. 1986). Finally, we assume that CS + US pairings can induce plasticity in the cerebellum and that the expression of CRs is mediated by increased activity in the appropriate sets of cells in the anterior interpositus nucleus of the cerebellum. Thus, the muscle groups involved in the CR are determined by the anterior interpositus nucleus neurons that are activated, and the magnitude of the response is determined by their degree of activity.

\section{SYNAPTIC ORGANIZATION OF THE CEREBELLUM}

The architecture of the cerebellum (Fig. 2)the cell types, their connectivity, and their synaptic physiology - is relatively well known (Eccles et al. 1967; Palay 1974; Chan-Palay 1977; Ito 1984). As noted above, inputs to the cerebellum are conveyed by two classes of afferents: the mossy fibers and climbing fibers. Mossy fibers originate from numerous sources throughout the brain, and different mossy fibers are activated by virtually every sensory modality, including a large number that receive input from the cerebral cortex. Mossy fibers branch profusely in the cerebellar cortex, make excitatory synapses with Golgi cells and a large number of granule cells, and also send excitatory collaterals to the cerebellar nucleus cells. Granule cell axons (parallel fibers) course through the folium making excitatory contacts with numerous Purkinje cells, which in turn inhibit the cells of the cerebellar nuclei. Thus, the mossy fibers can influence the output cells in the cerebellar nuclei both by direct excitatory projections and by the granule/Purkinje pathway in the cerebellar cortex. Climbing fibers arise from the IO nuclei and project directly to cerebellar Purkinje cells. In contrast to the extensive divergence of mossy fibers, each climbing fiber contacts $\sim 10$ Purkinje cells and each Purkinje cell receives input from only one climbing fiber.

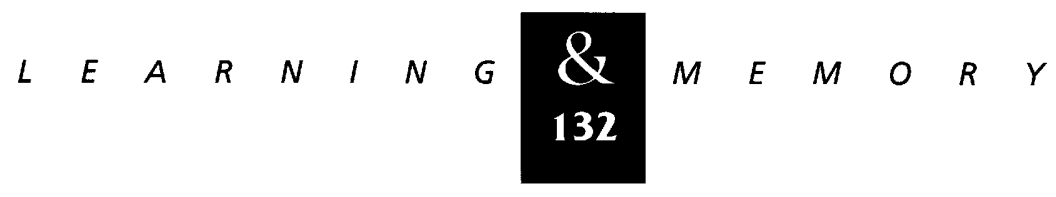


A

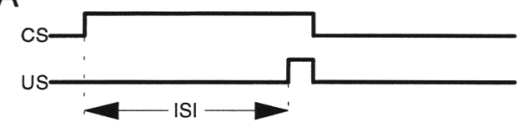

B
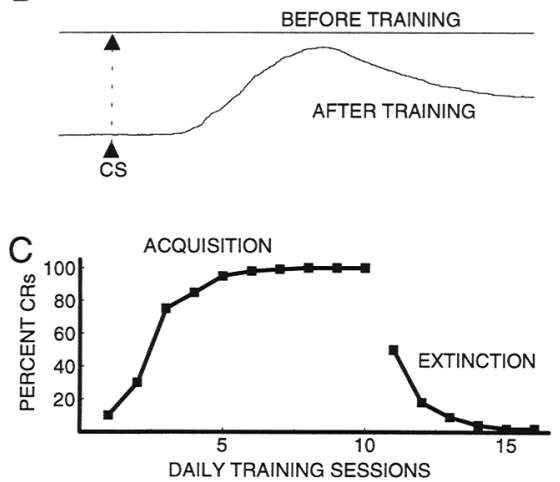

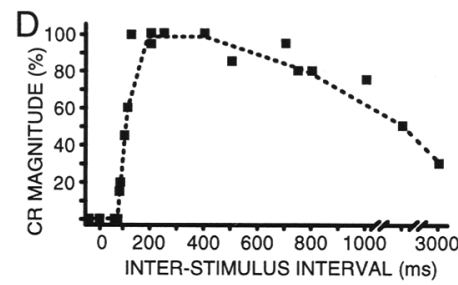

US
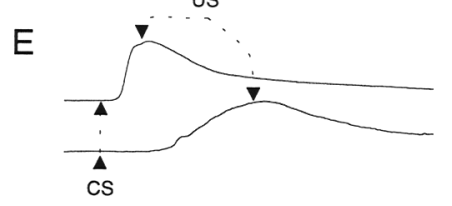

Figure 1: Behavioral properties of Pavlovian eyelid conditioning. (A) A schematic representation of a CS + US trial for a commonly used protocol (delay conditioning). The onset of the CS, a tone or a light, for example, precedes the onset of an US such as an air puff directed at the eye. The time between the onsets of the stimuli is the ISI. (B) Sample eyelid responses elicited by the presentation of a tone CS (onset depicted by the arrowheads). Before training there is no measurable eyelid response to the CS. After training the eyelid closes (upward deflection of the trace) in response to the $\mathrm{CS}$, and the response peaks near the normal time of US onset (not shown). (C) Sample acquisition and extinction curves showing the increase in response probability

during 10 days of CS + US training and the decline in response magnitude during 6 days of CS-alone extinction training. (D) The effects of the ISI on conditioning (the ISI function) taken from a number of published results (Schneiderman and Gormezano 1964; Schneiderman 1966; Smith 1968; Salafia et al. 1979). Each point represents the magnitude of conditioning for that ISI expressed as a percentage of the maximum responding observed in the particular paper. A negative ISI indicates the US precedes the CS, and a positive ISI indicates CS precedes the US. ( $E$ ) Two sample responses from animals trained with a relatively short (top) and relatively longer (bottom) ISI. CS onsets are indicated by upward pointing arrowheads, and the US onsets by downward arrowheads. The latencies to onset and rise-times of the responses vary systematically with the ISI such that the responses peak near US onset.

THE SEMINAL CEREBELLAR THEORIES OF MARR AND ALBUS

On the basis of this synaptic organization, Marr (1969) and, later, Albus (1971) formulated similar theories of cerebellar function. Marr suggested that the mossy fibers convey to the cerebellum information about the context in which movements occur. The representation of this context was said to be recoded through the divergent mossy fiber projections onto the very large population of granule cells. Marr suggested that movements in that context could be reinforced if climbing fiber inputs increase the strength of coactive $g r \rightarrow P k j$ synapses. Albus modified this theory by assuming that climbing fibers decrease the strength of coactive $g r \rightarrow P k j$ synapses. Many studies have since provided support for the basic architecture of the Marr/Albus theories and, in particular, for the plasticity rule proposed by Albus. For example, coactivation of climbing fiber and granule cell inputs to the Purkinje cells can decrease the strength of the $g r \rightarrow P k j$ synapses, a phenomenon called longterm depression (LTD) (Ito and Kano 1982; Ito et al. 1982; Ito 1988; Linden and Connor 1991; Linden et al. 1991; Schreurs and Alkon 1993, 1996).

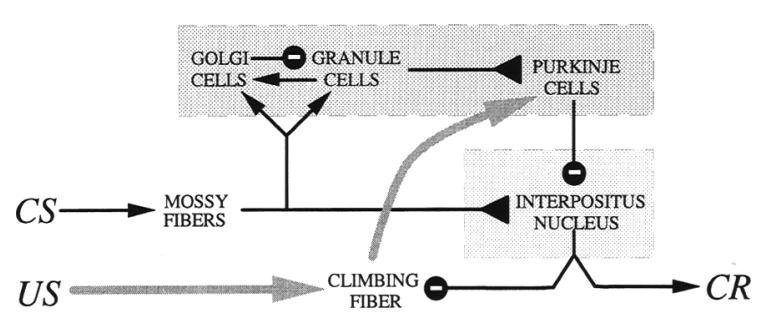

Figure 2: A schematic (and partial) representation of the synaptic organization of the cerebellum and its relation to the stimulus and response pathways involved in eyelid conditioning. The two classes of cerebellar afferents-mossy fibers and climbing fibers-are activated by the CS and US, respectively. Mossy fibers project directly to the output cells of the cerebellar nuclei and also to the granule and Golgi cells of the cerebellar cortex. The granule cells make excitatory synapses onto Purkinje cells that, in turn, inhibit the nucleus output cells. Granule cell activity in response to a particular mossy fiber input is influenced by interactions between the populations of granule and Golgi cells. In contrast, climbing fibers make spatially distributed connections onto a small number of Purkinje cells. The output cells of the cerebellar interpositus nucleus project to efferent pathways that are necessary for the expression of the conditioned eyelid responses. This output pathway also provides feedback inhibition of the climbing fibers.

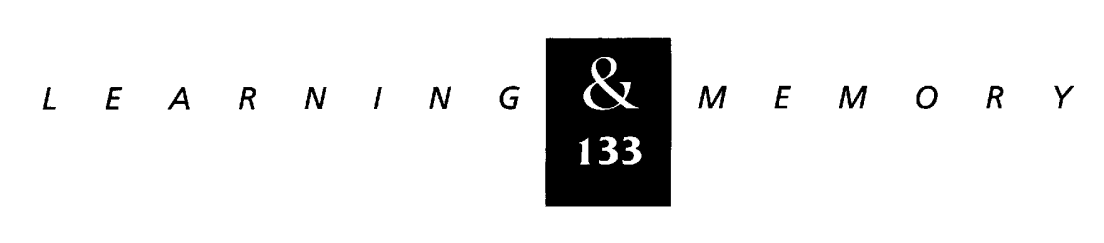


An important extension of this rule allows for $g r \rightarrow P k j$ synapses to increase in strength [i.e., to undergo long-term potentiation]. This assumption is based on experiments showing that $g r \rightarrow P k j$ synapses are potentiated if they are active in the absence of climbing fiber inputs (Sakurai 1987; Shibuki and Okada 1992; Schreurs and Alkon 1993; Salin et al. 1996). In addition, lesion studies examining eyelid conditioning and adaptation of vestibular reflexes (Robinson 1976; McCormick and Thompson 1984), as well as unit recordings during adaptation of wrist movements (Thach 1980; Thach et al. 1992), have produced results consistent with the spirit of the Marr and Albus theories. The present model is an elaboration of these theories.

\section{Hypothesis 1: Conditioning Involves Two Cerebellar Sites of Synaptic Plasticity}

We hypothesize that two sites of synaptic plasticity in the cerebellum are responsible for the acquisition and expression of conditioned eyelid responses: (1) the $g r \rightarrow P k j$ synapses in the cerebellar cortex and (2) the mossy fiber synapses in the interpositus nucleus ( $\mathrm{mf} \rightarrow \mathrm{nuc}$ ). Motor learningrelated plasticity at one or both of these sites has been proposed previously (Miles and Lisberger 1981; Ito 1982; Thompson 1986; Lisberger 1988; Perrett et al. 1993; Perrett and Mauk 1995; Raymond et al. 1996; Mauk 1997). The novel aspects of our hypothesis are found in the explicitly stated rules for plasticity at both sites.

\subsection{BIDIRECTIONAL PLASTICITY AT $g r \rightarrow P k j$ SYNAPSES IN THE CEREBELLAR CORTEX}

From the assumptions of Albus (1971) and subsequent empirical findings (Sakurai 1987; Ito 1988; Kano and Kato 1988; Linden and Connor 1991; Linden et al. 1991; Schreurs and Alkon 1993, 1996), we adopt the following bidirectional LTD/ LTP rule: $g r \rightarrow P k j$ synapses decrease in strength when active in the presence of a climbing fiber input (LTD) and increase in strength when active in the absence of a climbing fiber input (LTP). Although the exact timing required of the two inputs has not been precisely identified (Kano and Kato 1988; Chen and Thompson 1995; Schreurs and Alkon 1996), the model requires only that LTD occur when the inputs are active at approximately the same time.

\subsection{BIDIRECTIONAL PLASTICITY AT $\mathrm{mf} \rightarrow$ nuc SYNAPSES CONTROLLED BY PURKINJE CELL INPUTS}

At $\mathrm{mf} \rightarrow$ nuc synapses we assume a bidirectional plasticity that is dependent on the inhibitory Purkinje cell input: Synapses increase in strength $\left(\mathrm{LTP}_{\text {nuc }}\right)$ when active during transient decreases in Purkinje activity; that is, this rule is not engaged by the mere absence of Purkinje input. Conversely, we assume that $\mathrm{mf} \rightarrow$ nuc synapses decrease in strength $\left(\mathrm{LTD}_{\text {nuc }}\right)$ when active in the presence of strong inhibitory Purkinje cell input. We know of only one study in which plasticity in the nucleus was tested-high-frequency stimulation of mossy fibers enhanced a monosynaptic field potential recorded in cerebellar nuclei in vivo (Racine et al. 1986). Other, indirect evidence suggests that bidirectional changes in these synapses are important for adaptation of the vestibulo-ocular reflex (e.g., Miles and Lisberger 1981; Lisberger 1988) and for eyelid conditioning (Thompson 1986; Perrett et al. 1993; Perrett and Mauk 1995; Raymond et al. 1996; Mauk 1997). Thus, although experimental findings indirectly support the idea that $\mathrm{mf} \rightarrow$ nuc synapses are modifiable, the Purkinje-dependent rule for plasticity at $\mathrm{mf} \rightarrow$ nuc synapses is an assumption whose consequences we evaluate in the model.

\subsection{FACTORS INFLUENCING THE INDUCTION OF PLASTICITY}

The findings of Llinás and Mühlethaler (1988) suggest a concrete but speculative mechanism for inducing $\mathrm{LTP}_{\text {nuc }}$ and $\mathrm{LTD}_{\text {nuc }}$ at $\mathrm{mf} \rightarrow$ nuc synapses that is consistent with our assumptions. Recordings from cerebellar nucleus cells revealed a calcium conductance whose activation required depolarization from a hyperpolarized state, rather than depolarization from the resting potential. Given that Purkinje cells are normally active at high rates, it seems possible that this calcium conductance could be activated by transient decreases in the ongoing inhibitory input from Purkinje cells and that the resulting increase in intracellular calcium could induce LTP $_{\text {nuc }}$ in coactive $\mathrm{mf} \rightarrow$ nuc synapses. Drawing parallels with LTP and LTD in the hippocampus and neocortex (Artola et al.

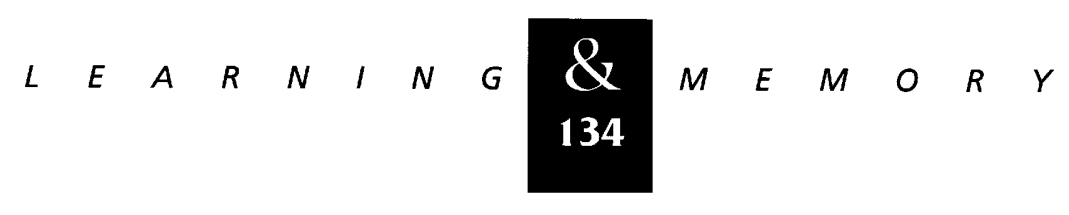


1990; Mulkey and Malenka 1992; Dudek and Bear 1993), $\mathrm{mf} \rightarrow$ nuc synapses may increase in strength when coactive during high levels of calcium and decrease in strength when active during lower levels of calcium, as may occur during strong inhibitory input from Purkinje cells (see Shibuki and Okada 1992).

Recordings have shown that Purkinje cells are normally active at high rates, and the corresponding tonic inhibition of nucleus cells highlights an important implication of our plasticity assumptions. Whereas LTD/LTP at the $g r \rightarrow P k j$ synapses depends on the patterns of stimuli conveyed by mossy fiber and climbing fiber inputs, LTP $_{\text {nuc }} /$ LTDnuc at the $\mathrm{mf} \rightarrow$ nuc synapses is controlled by mossy fiber collateral inputs and by inhibitory input from Purkinje cells. Thus, lesions of the cerebellar cortex should prevent further plasticity at $\mathrm{mf} \rightarrow$ nuc synapses. Without inhibition from Purkinje cells, our assumptions suggest that LTD $_{\text {nuc }}$ would not be possible. Similarly, without Purkinje cells there cannot be a transient release from inhi- bition that our assumptions require for the induction of LTP ${ }_{\text {nuc }}$.

\subsection{A TRIALS-LEVEL DESCRIPTION OF ACQUISITION, EXPRESSION, AND EXTINCTION OF CRs}

The above assumptions suggest the following trials-level account for cerebellar involvement in eyelid conditioning (Fig. 3). Prior to training, the mossy fibers activated by the CS only weakly excite the nucleus cells. This excitation is counteracted by the ongoing inhibitory input from Purkinje cells, which is not changed by the CS. Consequently, the initial acquisition of CRs results from the induction of LTD at $g r \rightarrow P k j$ synapses that are activated by the CS during a US-evoked climbing fiber input to the Purkinje cell. With repeated $\mathrm{CS}+\mathrm{US}$ pairings, the LTD at these $g r \rightarrow P k j$ synapses would decrease the average strength of the $g r \rightarrow P k j$ synapses active during the CS. Because

A Acquisition

1

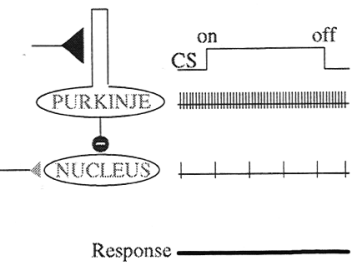

B Extinction

1

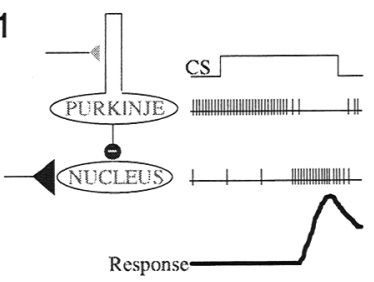

2

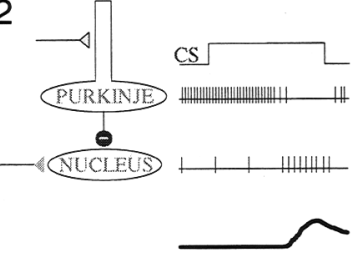

2

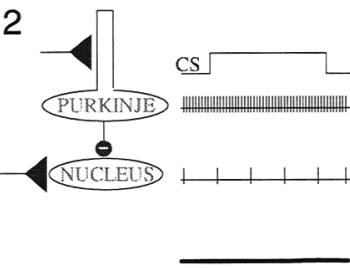

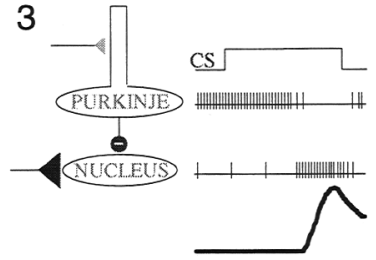

3

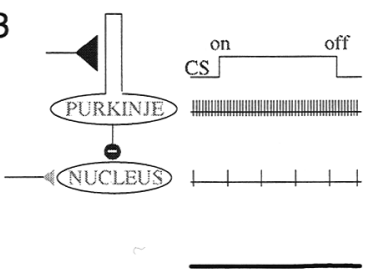

Figure 3: A schematic representation of the events hypothesized to be associated with the acquisition and extinction of CRs. Each of the six panels shows the granule cell synapses onto Purkinje cells ( $g r \rightarrow P k j$ ) and the mossy fiber synapses onto the nucleus cells ( $\mathrm{mf} \rightarrow$ nuc), where the relative strength of the synapse is indicated by the size and shading (stronger synapses are larger and darker). The right half of each panel shows the assumed activity of the Purkinje and nucleus cells during the presentation of a CS. The response that would be produced is shown schematically at bottom of each panel. (A) Acquisition: (1) presentation of a CS to an untrained animal; (2) early in training, LTD induced by paired presentation of CS + US has decreased the strength of the $g r \rightarrow P k j$ synapses; (3) with additional training, the induction of LTP at the $\mathrm{mf} \rightarrow$ nuc synapses combines with the learned pause in Purkinje activity to produce robust CRs. (B) Extinction: (1) At the beginning of extinction training, CRs are produced by plasticity in both the cerebellar cortex and cerebellar nucleus; (2) i.e., when the decline in CRs is nearly complete, the induction of LTP at $g r \rightarrow P k j$ synapses has restored robust Purkinje cell activity during the CS, preventing the expression of CRs despite the relative strength of the mf $\rightarrow$ nuc synapses; (3) at this point, presentation of additional CS alone extinction trials would induce LTD at $\mathrm{mf} \rightarrow$ nuc synapses.

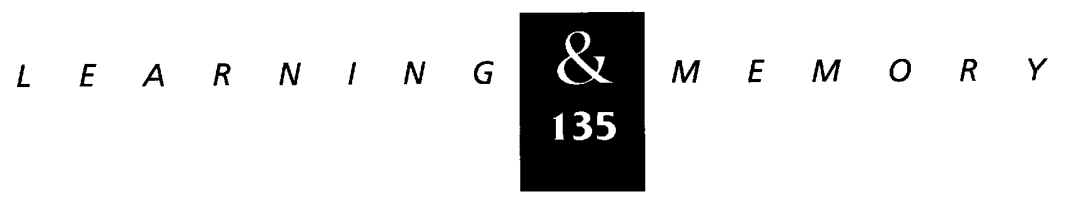


Purkinje cell activity presumably reflects the net effects of excitatory input from granule cells and ongoing inhibitory input from stellate and basket cells, training should lead to an acquired decrease in Purkinje activity and a phasic disinhibition of the interpositus cells during the CS (Fig. 3A, 1 and 2). This would have the dual effects of increasing the likelihood of eliciting CRs and inducing LTP $_{\text {nuc }}$ at CS-activated $\mathrm{mf} \rightarrow$ nuc synapses (Fig. 3A, 3), which would further increase the ability of the CS to generate a conditioned eyelid response.

In contrast, extinction can be explained by the LTP (reversal of LTD) induced at $g r \rightarrow P k j$ synapses during nonreinforcement of a trained CS, which results from CS activation of granule cells in the absence of climbing fiber input. This would eliminate the acquired pause in Purkinje activity, reinstate the inhibition of nucleus cells during the CS, diminish the CR, and promote the induction of LTD $_{\text {nuc }}$ (reverse LTP $_{\text {nuc }}$ ) at the $\mathrm{mf} \rightarrow$ nuc synapses (Fig. 3B, 1-3). These changes at $g r \rightarrow P k j$ synapses in the cortex and $\mathrm{mf} \rightarrow$ nuc synapses in the nucleus would each act to diminish the ability of the CS to elicit CRs.

Thus, for both acquisition and extinction, our assumptions predict that plasticity is first induced in the cortex at $g r \rightarrow P k j$ synapses; this, in turn, allows subsequent training trials to induce changes in the opposite direction at $\mathrm{mf} \rightarrow$ nuc synapses. Because Purkinje cells inhibit nucleus cells, decremental changes in cortical synapses and incremental changes in nucleus synapses work together to increase the magnitude of the $\mathrm{CR}$, whereas incremental changes in the cortex and decremental changes in the nucleus lead to response extinction.

\section{Hypothesis 2: Climbing Fiber Activity Is Self-Regulated to an Equilibrium Level}

A tacit assumption common to most models of learning is that in the absence of the CS, US, or context cues associated with the US, there are no opportunities for reinforcement or nonreinforcement. Similarly, most neural networks permit plasticity only during training trials and not, for example, between trials or during probe trials. In contrast, realistic neural models of learning must confront the constraints that (1) the neurons whose activity represents the CS and the US can be active in the intervals between training trials or training sessions and (2) the rules for plasticity can- not be arbitrarily disengaged during these times. With the cerebellum, for example, climbing fibers display low and fairly regular rates of spontaneous activity (see Keating and Thach 1995). Moreover, it seems implausible that the mossy fiber and granule cells that encode the CS are always silent in the absence of the CS. This would impose the equally unrealistic requirement that every stimulus is encoded by a unique subset of mossy fibers and granule cells that are active if (and only if) the stimulus is present. These issues pose difficult questions. For example, what prevents the fortuitous pairings of granule cell and climbing fiber activity from inducing LTD at $g r \rightarrow P k j$ synapses to the point of saturation? How can 100 US-evoked climbing fiber inputs during a training session convey information against a background of 3600 spontaneous climbing fiber inputs during the same period and $>80,000$ before the next session? Moreover, if the granule cells that encode a CS are also occasionally active in the absence of the CS or US, what prevents spontaneous extinction of CRs between sessions?

This section describes a two-part hypothesis to address these issues. First, the combination of spontaneous mossy and climbing fiber activity suggests that plasticity at $g r \rightarrow P k j$ synapses is best understood as a stochastic process in which net changes in strength depend on the probability that synapse activation is paired with a climbing fiber input. Second, we propose that the inhibitory pathways from cerebellar nuclei to climbing fiber cell bodies in the IO (Groenewegen and Voogd 1977; Groenewegen et al. 1979; Oscarsson 1980; Houk and Gibson 1986; Anderson et al. 1988; Angaut and Sotelo 1989; de Zeeuw et al. 1989; Ruigrok and Voogd 1990) provide a form of negative feedback that balances the likelihood of LTD and LTP events such that the net strength of $g r \rightarrow P k j$ synapses remains relatively constant under all but two conditions: when a CS occurs with an unexpected US-as during acquisition-and when a CS signals the US, but the US is omitted-as during extinction.

\subsection{PROBABILISTIC LTD/LTP AND EQUILIBRIUM CLIMBING FIBER ACTIVITY}

Bidirectional LTD/LTP implies that $g r \rightarrow P k j$ synapses will undergo plasticity whenever they are active ("active" might mean one spike or a burst of spikes); they undergo LTD when there are chance

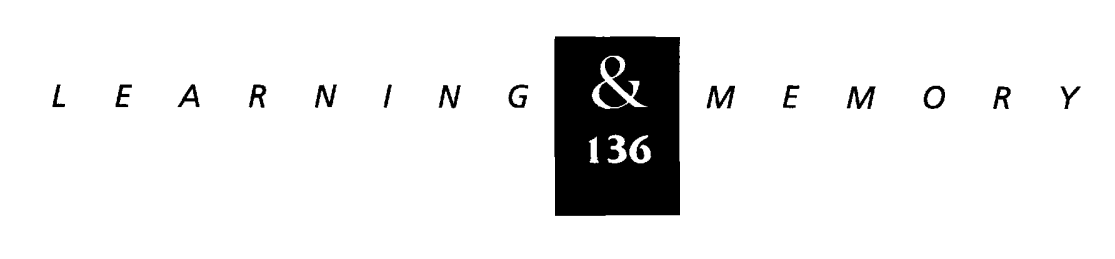


pairings of granule cell and climbing fiber activity and undergo LTP when granule cell activity occurs alone. Thus, the rate of net change of a $g r \rightarrow P k j$ synapse will depend on its activity, and the expected direction of change will depend on the probability that its activity is paired with a climbing fiber input. When the probability of pairing is high, the expected result is a net decrease in synaptic strength (LTD), whereas a net increase (LTP) is expected when the pairing probability is low (i.e., granule cell activity in the absence of a climbing fiber input). Between these two extremes there exists an intermediate probability where the effects of LTD and LTP balance and the expected net change in synaptic strength is zero. At this level of climbing fiber activity, any single instance of synapse activity will result in an increment or decrement in strength, but these changes occur in proportions for which the expected net change is zero. For convenience we refer to this equilibrium level of climbing fiber activity as $C F^{E q}$.

The ability to maintain the spontaneous climbing fiber activity at $C F^{E q}$ could serve an important role in maintaining a relatively constant pattern of synaptic weights as established by previous motor learning experience. Outside of the conditioning context, any $g r \rightarrow P k j$ synaptic activity would be equally likely to promote an increase or decrease in strength, with the expected net change being zero. However, even slight deviations from $C F^{E q}$, if persistent, would cause the strength of all $g r \rightarrow P k j$ synapses to drift and to eventually saturate at their minimum or maximum values.

To regulate climbing fiber activity with sufficient precision to achieve stability would appear to require coupling the average $g r \rightarrow P k j$ synaptic weight, as indexed by activity of the Purkinje cells, to the modulation of climbing fiber activity by negative feedback. A central idea we advance here is that the inhibition of climbing fibers by cerebellar output (Fig. 2) represents this type of negative feedback and could regulate spontaneous climbing fiber activity to $C F^{E q}$. To illustrate, consider the consequences of spontaneous climbing fiber activity above $C F^{E q}$ (Fig. 4A). Chance pairings would occur too frequently in that LTD would prevail over LTP, resulting in a net decrease in synaptic strengths. The decrease in Purkinje cell activity would increase the activity of cerebellar nucleus cells through reduced inhibition from Purkinje cells. The increased inhibitory output from the nucleus cells would, in turn, cause a corresponding decrease in the activity of climbing fibers.
Thus, the expected consequence of excess climbing fiber activity is an increased inhibition of climbing fibers resulting from a net decrease in the strength of the $g r \rightarrow P k j$ synapses. Inappropriately low rates of spontaneous climbing fiber activity could be similarly counteracted by an inverse series of events. Consequently, climbing fiber activity could be driven to a level at which LTD and LTP events balance and the expected net change in $g r \rightarrow P k j$ synapses is zero. A recent mathematical analysis supports these ideas and suggests that under these conditions LTP and LTD at the $\mathrm{mf} \rightarrow$ nuc synapses also balance (G.T. Kenyon, J.F. Medina, and M.D. Mauk, in prep.).

\subsection{THE CONSEQUENCES OF PROBABILISTC PAIRINGS OF MOSSY FIBER AND CLIMBING FIBER INPUTS}

We can now elaborate on the previous trialslevel description of acquisition, retention, and extinction of CRs by considering the consequences of presenting a CS under a variety of conditions. The left ordinate of Figure $4 \mathrm{~B}$ shows the probability that activity of a $g r \rightarrow P k j$ synapse is paired with a climbing fiber input, the right ordinate shows the corresponding change in synaptic strength under bidirectional LTD/LTP rules, and the abscissa represents time. The horizontal boundry between the light and dark gray in the graph depicts an arbitrarily chosen value for $C F^{E q}$. In an untrained animal, because the $g r \rightarrow P k j$ synapses activated by a CS-alone trial should, like all other $g r \rightarrow P k j$ synapses, be reinforced at $C F^{E q}$ the expected net change in their synaptic weights should be zero (Fig. $4 \mathrm{~B}, 1$ ). When this same CS is paired with a US, the probability of pairing increases during the trial above that produced by $C F^{E q}$, owing to the excitatory input to the climbing fibers added by the US. The expected results are a net decrement in strength of the $g r \rightarrow P k j$ synapses activated by the CS and acquisition of CRs (Fig. 4B, 2, and C). As acquisition proceeds, the CS would exhibit an increased ability to elicit eyelid responses and to inhibit climbing fibers. This would diminish the ability of the US to activate climbing fibers and return the probability of pairing during the CS + US trial to that produced by $C F^{E q}$. At this point the excitatory input to the climbing fibers from the US is exactly counteracted by CR-related inhibition of the climbing fibers.

Figure $4 \mathrm{~B}$ (3) illustrates how the modulation of

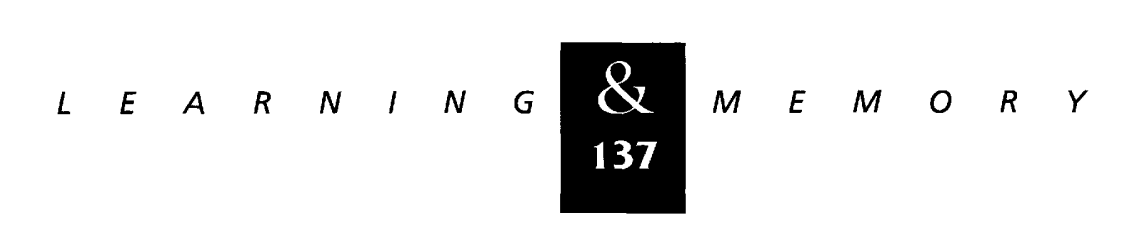


climbing fibers could be important for extinction as well. During an unreinforced CS, climbing fiber activity should fall below $C F^{E q}$ owing to the CRrelated inhibition of climbing fibers that is now unopposed by the excitatory drive provided by the US. Here, the expected change in synaptic strength is a net increase, which would increase Purkinje activity during the CS, restore inhibition of nucleus cells during the $\mathrm{CS}$, and produce extinction of CRs (Fig. 4C). The CS-generated inhibition of climbing fibers would decrease as the CRs diminish over the course of extinction training, and the expected changes in $g r \rightarrow P k j$ synaptic strength should again be zero when the probability of pairing returns to that produced by $C F^{E q}$. Thus, the result of either acquisition or extinction is that a set of synaptic weights changes such that climbing fiber equilibrium is achieved in the presence of background cues alone and during the CS. Moreover, under our assumptions, the induction of plasticity at $\mathrm{mf} \rightarrow$ nuc synapses requires the initial induction of plasticity at $g r \rightarrow P k j$ synapses. Consequently, the above arguments for $g r \rightarrow P k j$ synapses apply indirectly for $\mathrm{mf} \rightarrow$ nuc synapses as well.
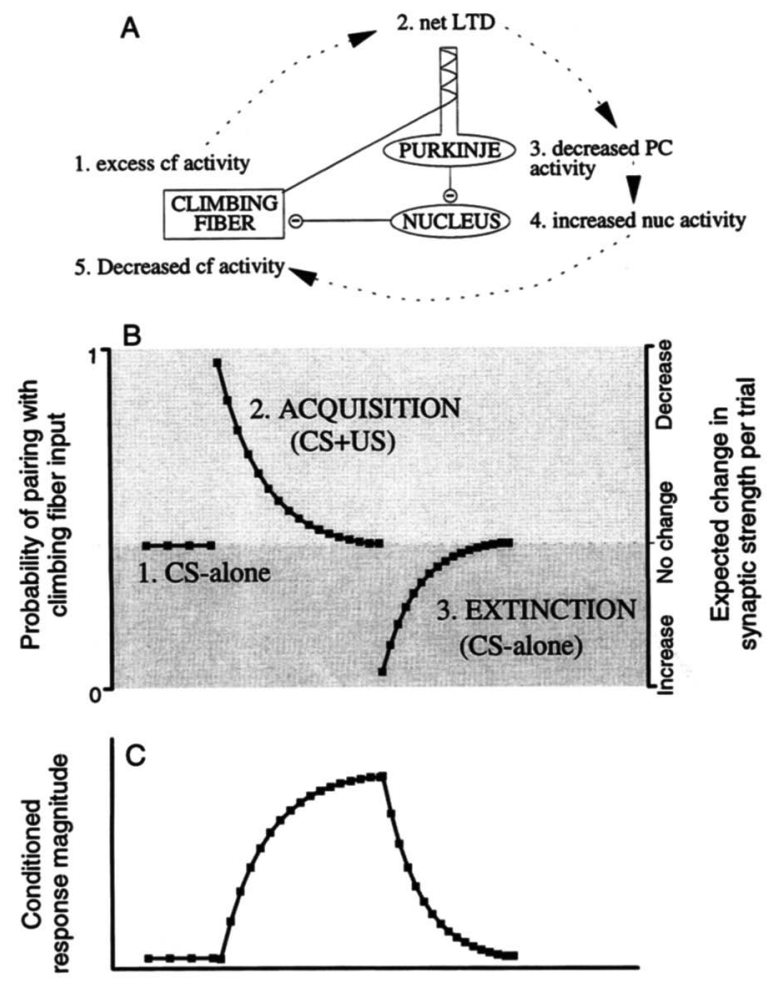

\subsection{CS + US TRIALS PROMOTE TWO PHASES OF SYNAPTIC PLASTICITY}

Here, we extend the trials-level description further by distinguishing between events that are initiated during the trial from those that occur afterwards. The need for this distinction arises from the prediction, implied by an equilibrium level of climbing fiber activity, that each CS + US trial initiates two phases of plasticity (Fig. 5). One phase involves the LTD induced during the trial at $g r \rightarrow P k j$ synapses activated by the CS. Because this LTD produces a net decrease in the synaptic input to the Purkinje cell, climbing fiber activity will decrease below $C F^{E q}$. Thus, during a period following the trial in which climbing fiber activity remains depressed, there will be a second phase of plasticity in which active $g r \rightarrow P k j$ synapses undergo more LTP than LTD until climbing fiber equilibrium is restored. Each $g r \rightarrow P k j$ synapse will undergo this net increase in strength proportional to its background activity following the trial. Thus, the expected net change in each $g r \rightarrow P k j$ synapse after a CS + US trial and after the subsequent return

Figure 4: The self-regulating equilibrium of climbing fiber activity and its influence on acquisition and extinction. $(A)$ The pathways involved in the feedback regulation of climbing fiber activity to the equilibrium level at which the expected net change in $\mathrm{gr} \rightarrow P k j$ synapses is zero. This example begins with a level of climbing fiber activity above equilibrium (1). This produces more LTD events than LTP, and the $g r \rightarrow P k j$ synaptic weights gradually decrease (2). This decreases Purkinje cell activity (3), which decreases inhibition and increases the activity of nucleus cells (4). The resulting increase in inhibitory input brings climbing fiber activity back down to the equilibrium level. (B) The effect of self-regulating climbing fiber equilibrium on acquisition and extinction. The ordinate at left shows the probability than any given activation of a $g r \rightarrow P k j$ synapse is paired with climbing fiber activity. The ordinate at right shows the corresponding net effect on the strength of $g r \rightarrow P k j$ synapses. The horizontal line indicates the equilibrium level of climbing fiber activity at which LTD and LTP balance. Because climbing fiber activity is regulated to this level, the $g r \rightarrow P k j$ synapses activated by a CS in an untrained level show no change (1). The presentation of a CS + US trial activates those $g r \rightarrow P k j$ synapses when the probability of climbing fiber activity is much higher owing to the US (2). As acquisition proceeds, the response-related inhibition of climbing fibers counteracts the US-evoked excitation of climbing fibers, and the probability that the $g r \rightarrow P k j$ synaptic activity associated with CS will be paired with a climbing fiber input decreases to the equilibrium level. During CS-alone extinction training, climbing fiber activity during the CS falls below the equilibrium level owing to the response-related inhibition that is unopposed by excitation from the US (3). As CRs decline, so does the response-related inhibition of climbing fibers, and thus the climbing fiber activity during the trial gradually returns to the equilibrium level.

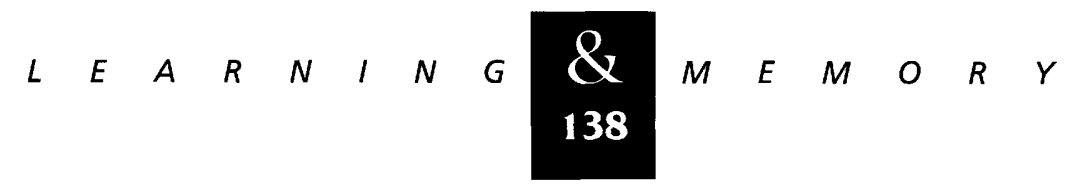




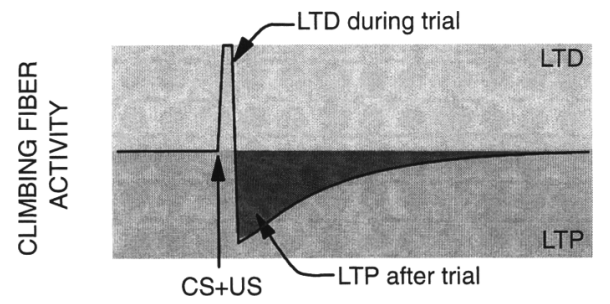

Figure 5: The two phases of plasticity induced by a CS + US trial that are suggested by a self-regulating equilibrium of climbing fiber activity. The ordinate depicts climbing fiber activity, and the abscissa depicts time. As in Fig. 4B, the horizontal line represents the equilibrium level of climbing fiber activity. Before the trial, spontaneous climbing fiber activity is in equilibrium. During the CS + US trial, the US increases climbing fiber activity above equilibrium and the $g r \rightarrow P k j$ synapses activated by the CS undergo LTD. This decreases Purkinje (and thus climbing fiber) activity below equilibrium. Therefore, a second phase of plasticity occurs after the trial, during which $g r \rightarrow P k j$ synapses tend to undergo LTP with a frequency proportional to their background activity.

to equilibrium should be proportional to the difference between its activity when the US-elicited climbing fiber input arrives during the CS (which produces LTD) and its background level of activity after the trial (which produces LTP). Synapses that are more active than normal when the US is presented undergo more LTD than LTP and display a net decrease in strength. The opposite holds for synapses that are less active than normal during the CS. It is important to note that this second phase of plasticity is attributable entirely to factors that maintain climbing fiber equilibrium and is not a direct product of post-trial US processing.

\section{Hypothesis 3: A Time-Varying Granule Cell Representation of the CS Mediates CR Timing}

In the next two sections we expand the trialslevel description to include real-time, ISI-related phenomena. First, we address the ISI-dependent timing of CRs by hypothesizing a cerebellar mechanism that produces a time-varying representation of the CS. In the subsequent section we suggest how this mechanism may also provide an account for the relationship between rate of learning and the ISI (i.e., the ISI function).

\subsection{A GRANULE CELL CS-REPRESENTATION THAT PROVIDES TEMPORAL DISCRIMINATION}

The ISI not only determines whether CRs are acquired but also influences their timing (Schneiderman and Gormezano 1964; Schneiderman 1966; Frey and Ross 1968; Smith et al. 1969). Several studies have provided evidence that response timing cannot be mediated by a unitary, time-invariant CS representation. For example, Millenson et al. (1977) showed that when rabbits were trained using a single CS that was reinforced at two ISIs (e.g., the ISI was $200 \mathrm{msec}$ for half the trials and was $700 \mathrm{msec}$ for the remaining trials), they acquired CRs with two peaks, each peak corresponding to one of the training ISIs. Clearly, these data require an ability to discriminate different times during the $\mathrm{CS}$, that is, that the $\mathrm{CS}$ representation consists of a time-varying series of discriminable features that can be differentially reinforced (Millenson et al. 1977; Mauk and Ruiz 1992; Perrett et al. 1993). We address these requirements by proposing that different subsets of granule cells become active at different times during the CS and that this provides the temporal discrimination necessary for CR timing.

In Figure $6 \mathrm{~B}$, a generic form of the proposed time-varying granule cell representation of a CS is contrasted with a time invariant representation (Fig. 6A). In both panels, the ordinate represents a hypothetical population of granule cells and the abscissa represents time during a CS. Because the time-invariant CS representation is similar at different times during the stimulus, it provides no basis for temporal coding. Training at any effective ISI would modify the same synapses. This would preclude the possibility of producing double peaked responses with training using two ISIs. Moreover, because these modified $g r \rightarrow P k j$ synapses are active soon after CS onset, the CRs should show relatively short latencies to onset. These onset latencies could vary with the ISI only to the extent that associative strength in the nucleus can influence the rise time to the threshold for eliciting a response. In contrast, a time-varying representation as depicted in Figure 6B would encode not just the presence of the CS, but could encode different temporal segments of the CS. CS + US pairings with a particular ISI would modify a subset of $g r \rightarrow P k j$ synapses whose activation occurs near US onset, because both the acquired decrease in Purkinje activity during the CS and the associated CRs would be delayed until just before US onset

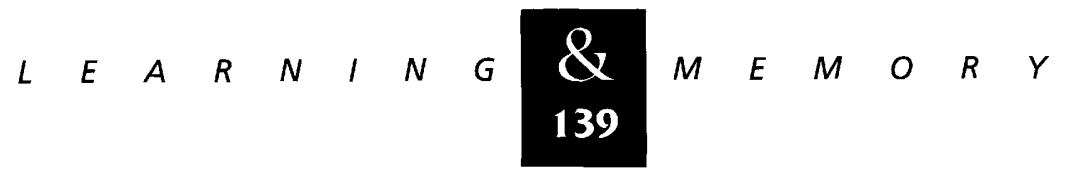


A

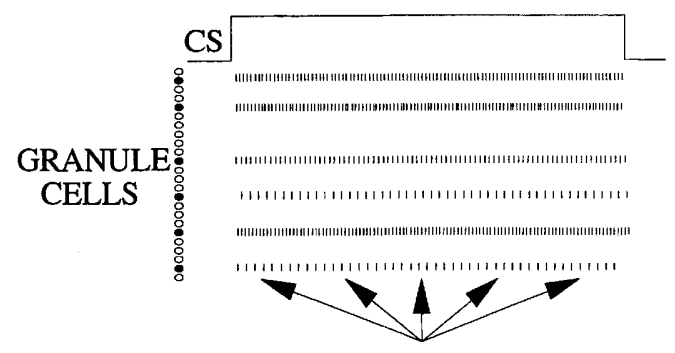

No Temporal

discrimination
B

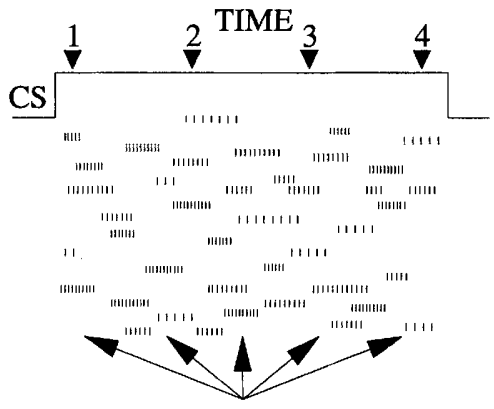

Temporal discrimination

Figure 6: Time-invariant and time-varying granule cell activity during a CS. Both panels show hypothetical raster plots of the activity of a population of granule cells (ordinate) as a function of time during the presentation of a CS (abscissa). (A) A time-invariant representation of the CS in which a set subset of granule cells is activated throughout the extent of the stimulus. This representation provides no temporal discrimination. Training at any ISI would produce responses with the same short latency to onset and to peak. (B) A time-varying representation in which different subsets of granule cells become active at different times during the CS. Reinforcing this representation at a particular ISI would produce responses timed to peak near US onset.

when the greatest proportion of "conditioned" $g r \rightarrow P k j$ synapses would be active. This type of CS representation could mediate the kinds of response timing that have been observed in the literature, such as double peaked responses with training using a single CS and two training ISIs.

\subsection{A MECHANISM FOR TIME-VARYING ACTIVATION OF GRANULE CELLS}

We also propose that the inhibitory influence of Golgi cells is a key factor in the production of a time-varying granule cell representation of CSs. If granule cells received input only from mossy fibers, then the subsets active during a CS could vary only to the extent that the mossy fiber activity varies during the CS (see Footnote 1). However, granule cells are also inhibited by Golgi cells, which receive excitatory input from both mossy fibers and granule cells (see Fig. 2). This synaptic arrangement suggests that the Golgi cells allow the ongoing activation of granule cells (by mossy fibers) to be modulated by previous activity of other mossy fibers and granule cells (see Fig. 6B). Thus, the granule cell activity at any time helps shape which granule cells will be activated next. For example, the granule cell activity at time " 1 " in Figure 6B would help determine the activity at time " 2 ." Likewise, time " 2 " activity would influence subsequent activity at time " 3 ," and so on. This would produce a characteristic variation in the granule cell activity throughout a stimulus.

\section{Hypothesis 4: Across-Trials Consistency of the CS Representation Determines the ISI Function}

In addressing the relationship between the ISI and rate of acquisition, most theories have adopted assumptions similar to those proposed by Hull (1943). (1) A CS activates a characteristic neural representation whose strength varies as a function of CS duration or trace interval, (2) the ability of the US to modify synapses will be proportional to the strength of the CS trace at the time of reinforcement, and, thus, (3) variation of the trace strength during the CS determines the ISI function. Here, we advance an hypothesis that is somewhat different than Hull's: The ability of granule cell activity to provide a consistent representation of the CS from one trial to the next (across-trials consistency) varies throughout the duration of the CS. We propose that the across-trials consistency of the activity representing the CS is an inverted $\mathrm{U}$ shaped function of the time from CS onset.

\subsection{THE NEED FOR ACROSS-TRIALS CONSISTENCY IN THE CS REPRESENTATION}

Conditioning requires a degree of across-trials consistency in the CS representation because a CS can only elicit CRs if it activates, within a narrow window of time, a sufficient number of the synapses that were modified by previous CS + US pairings. Given our assumption that the CS is repre-

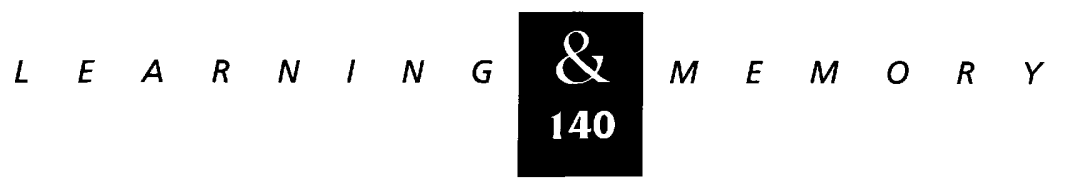


sented as a sequence of granule cell subsets active at different times during the stimulus, it is necessary that the ordering of this sequence be consistent from one trial to the next. Specifically, the time-varying pattern of granule cell activity, as schematized in Figure 6B, must be consistent from one trial to the next over the range of ISIs effective in producing conditioning. Consequently, decreases in across-trials consistency of granule activity should degrade the rate of conditioning, and variations in across-trials consistency for different segments of the CS would result in different rates of learning for different ISIs.

\subsection{THE INFLUENCE OF NOISE ON THE TIME-VARYING CS REPRESENTATION}

As a useful starting point, we assume that in the absence of the CS (i.e., during the inter-trial interval) there is a relative lack of consistency in the pattern of granule cell activity, despite the static contextual stimuli that may be represented by characteristic mossy fiber activity. This lack of consistency in the granule cell activity between trials would be responsible for the fact that USalone presentations do not promote acquisition of conditioned eyelid responses to the context. Although the climbing fiber inputs activated by the US would induce LTD in $g r \rightarrow P k j$ synapses that happen to be active at the time, substantially different subsets of synapses would be modified each trial. Moreover, the induction of LTD at these synapses would move climbing fiber activity below the equilibrium level, and all synapses would subsequently be expected to undergo LTP proportional to their background level of activity. The critical point is that without across-trials consistency each synapse's background activity determines both its likelihood of undergoing LTD (during the US) and undergoing LTP (as equilibrium is restored). Because each synapse would be as likely to decrease as increase in strength, no net synaptic changes should occur. In our view, the fact that the CS must precede the US by $-70-80 \mathrm{msec}$ for conditioning to occur (Fig. 1D) may reflect the CS exposure required to promote sufficient levels of across-trials consistency in the granule cell activity. Similarly, optimal ISIs represent the amount of CS exposure required to produce the highest levels of consistency across trials (Fig. 7).

Why then does the ability of the CS to support conditioning decline as the ISI increases? This may
A

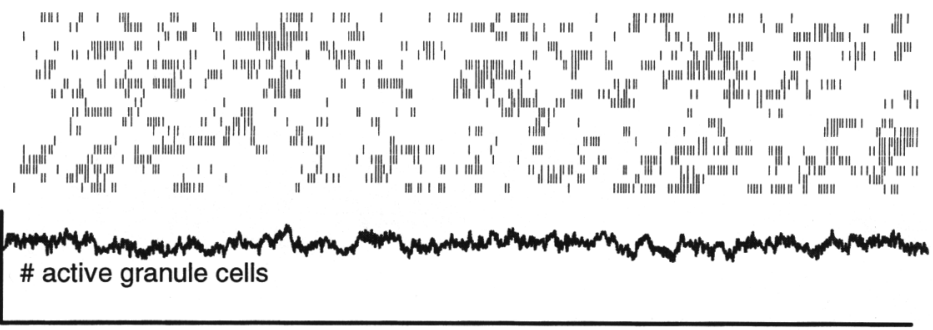

B
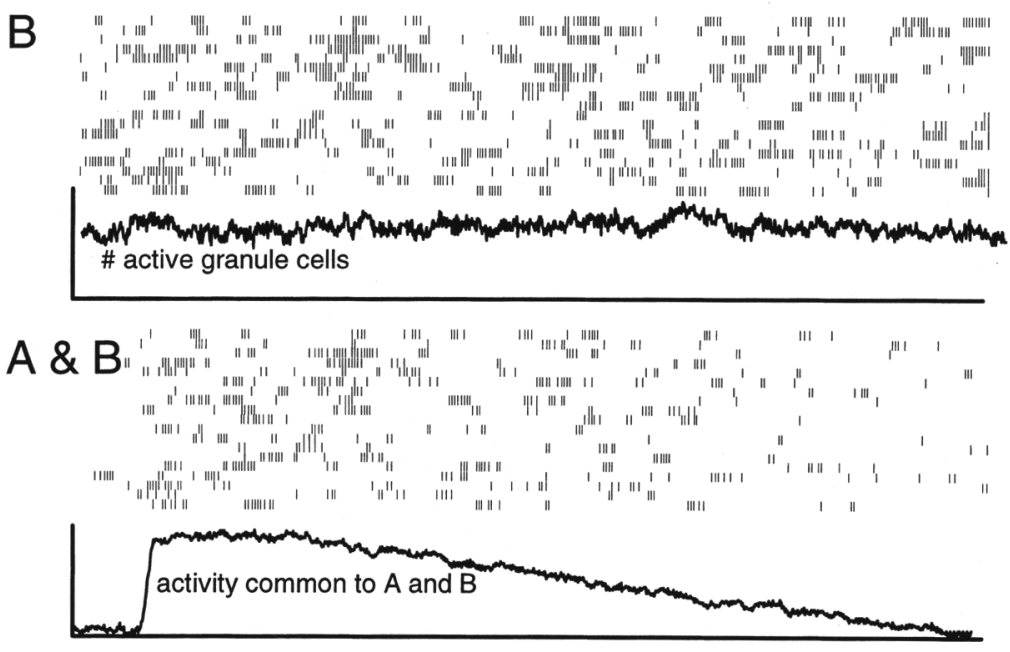

Figure 7: A schematic representation of across-trials consistency in the activity of granule cells activated by the CS. The two panels at top show hypothetical raster plots of granule cell activity as a function of time during the presentation of the same CS. For both panels the graph depicts a measure of the total amount of granule cell activity, which does not change appreciably during the CS. The third panel $(A \& B)$ shows the granule cell activity that is common to both trials, which is a measure of the acrosstrials consistency of granule activity. The graph associated with the panel at bottom shows that the common activity or acrosstrials consistency first increases then decreases during the CS. We propose that this across-trials consistency contributes to the conditionability of the CS at each time and therefore helps produce the ISI function.

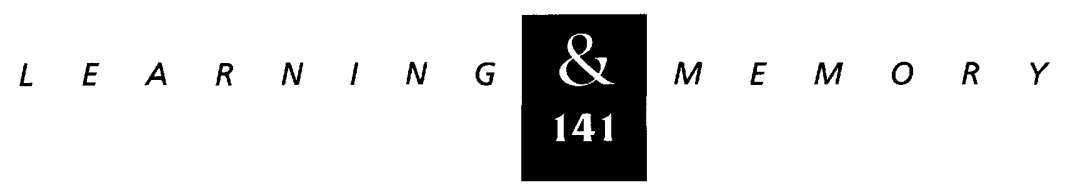


reflect an inherent tendency for small amounts of inconsistency early in the CS to be amplified into large amounts of inconsistency in later portions of the CS (see Lorenz 1963). This tendency could arise from the way in which the activity of each cell at any given time is determined by previous activity in many other cells. A spurious spike in a granule cell may subsequently lead to spurious activity in Golgi cells that, given the divergence of their projections, could then lead to even more spurious activity (or lack of activity) in other granule cells. Thus, the degradation in consistency produced by a uniform noise source can accumulate and lead to a time-dependent decrease in the across-trials consistency of the neurons activated by a CS. The sources of noise could be as subtle as the statistical nature of transmitter release and ion channel openings/closings. In this view, long ISIs are ineffective for the same reasons that repeated US-alone trials do not support eyelid conditioning to the context; the US modifies the subset of $g r \rightarrow P k j$ synapses that happens to be active, but these subsets are sufficiently different from trial to trial to prevent the development of a CR.

\section{Accounts of Training and Physiological Manipulations on Behavioral Outcomes}

We have proposed four interrelated hypotheses regarding cerebellar mechanisms of eyelid conditioning, which are summarized schematically in Figure 8. In this section we show how the assumptions interact to provide detailed accounts for a wide range of eyelid conditioning.

\section{ACQUISITION, EXTINCTION, AND CLIMBING FIBER EQUILIBRIUM}

Together, the four hypotheses imply that eyelid conditioning is mediated by interactions between (1) the rules for inducing synaptic plasticity at two cerebellar sites (cortex and nuclei), (2) the factors influencing the activity of climbing fibers, and (3) the nature of the CS representation in the cerebellum. Within the context of our assumptions, the cerebellum is a self-regulating system that maintains an equilibrium at which synapses are often modified, but the net change in strength of each synapse is zero. Pavlovian conditioning procedures can activate cerebellar afferents in ways that disturb this equilibrium and induce synaptic weight changes that mediate CRs.

Although theories of Pavlovian conditioning generally focus on events that occur during the CS + US trial, the present model suggests that it is also important to consider the equilibrium that exists before training and the events that occur after each trial. The activity of cerebellar inputs and cerebellar neurons elicited by training trials occurs against a background of ongoing mossy fiber and granule cell activity and an equilibrium level of climbing fiber activity $\left(C F^{E q}\right)$. Paired CS + US trials can provide conditions that disrupt this equilibrium and promote acquisition of CRs. These conditions are (1) repeated, US-evoked increases in climbing fiber activity above $C F^{E q}$, during (2) CSevoked granule cell activity that consistently differs from its background level. Extinction of CRs also occurs owing to a return to climbing fiber equilibrium. During the presentation of a CS-alone trial to a trained animal, the CR-related inhibition of climbing fibers, which is now unopposed by excitatory input from the US, brings the spontaneous climbing fiber activity below $C F^{E q}$. This leads to net LTP at $g r \rightarrow P k j$ synapses and the gradual decrease in the pause in Purkinje cell activity during the CS.

In the preceding sections, we have shown how the model can account for simple acquisition and extinction of CRs, as well as for the existence of spontaneous climbing fiber activity. We now explore additional and more detailed predictions of the model.

\section{RELATIVE DISTRIBUTION OF PLASTICITY BETWEEN CORTEX AND NUCLEUS}

Our assumptions imply that the relative distribution of plasticity can vary as a function of the amount and type of training (Mauk 1997). During acquisition, the paired presentation of CS and US would first induce LTD at the CS-activated $g r \rightarrow P k j$ synapses. The resulting training-induced pause in Purkinje activity during the CS would contribute both to the expression of CRs and to the induction of LTP ${ }_{\text {nuc }}$ at CS-activated $\mathrm{mf} \rightarrow$ nuc synapses. Our assumptions therefore permit, but do not demand, that CRs could appear before significant plasticity at $\mathrm{mf} \rightarrow$ nuc synapses occurs. However, with continued training, responses would be mediated by plasticity in both the cortex and nucleus. This suggests that the degree to which CRs are retained following lesions of the cerebellar

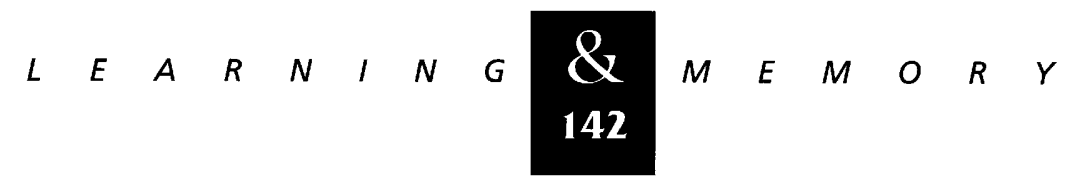


Figure 8: A schematic summary of the four main hypotheses that comprise the model. We propose: (1a) Plasticity at $g r \rightarrow P k j$ synapses is guided by climbing fiber inputsynapses active during a climbing fiber input decrease in strength, and those active without a climbing fiber input increase in strength; ( $1 b$ ) plasticity at $\mathrm{mf} \rightarrow$ nuc synapses is guided by inhibitory input from Purkinje cells-synapses increase in strength when active during transient decreases in the ongoing Purkinje input and decrease in strength when active during strong Purkinje input; (2) inhibition of climbing fibers by cerebellar output helps regulate spontaneous climbing fiber activity to an equilibrium level at which LTD and LTP balance and the expected net change in $\mathrm{gr} \rightarrow P$ j synapses is zero; (3) interactions between the populations of granule and Golgi cells help convert temporally constant mossy fiber inputs into time-varying activation of different granule cells at different times during a stimulus (a CS); and (4) these same interactions confer a sensitivity to noise such that the across-trials consistency of the granule cells activated by a CS first increases and then gradually fades during the stimulus. This variation in across-trials consistency influences learning and in part mediates the ISI function.

cortex may depend on the amount of training. Early in training, before the induction of significant plasticity at $\mathrm{mf} \rightarrow$ nuc synapses, a lesion might abolish the expression of CRs, whereas later in training, lesions should spare retention of (improperly timed) CRs. This training-dependent distribution of plasticity may in part explain the variable degrees of retention that have been seen following lesions of the cerebellar cortex.

The relative distribution of plasticity between the cerebellar cortex and nucleus may be sensitive to the type of training as well. Starting with Marr's (1969) notion that mossy fiber inputs to the large population of granule cells is an expansion recoder, that is, a device that increases discriminability of different stimuli, we can view the populations of mossy fibers and of granule cells as providing relatively coarse and fine CS representations, respectively. Fairly similar mossy fiber inputs would give rise to less similar granule cell representations. Thus, we can consider a functional stimulus representation unit as being comprised of a small number of $\mathrm{mf} \rightarrow$ nuc synapses and a much larger population of $g r \rightarrow P k j$ subsets that can be active at the same time. As the coarser, lower resolution representation, the $\mathrm{mf} \rightarrow$ nuc synapses might be activated by a relatively wide range of stimuli, for example, tones. The higher resolution representation provided by the subsets of $g r \rightarrow P k j$ synapses might code a more restricted range of auditory frequencies as well as different temporal segments of the same auditory stimulus.

Given this arrangement, the strength of $\mathrm{mf} \rightarrow$ nuc synapses may reflect the average CR coded by each $g r \rightarrow P k j$ synapse, weighted by its frequency of activation. This follows from the notion that $\mathrm{mf} \rightarrow$ nuc synapses increase in strength when they are coactive with a subset of $g r \rightarrow P k j$ synapses that code for a CR. Conversely, $\mathrm{mf} \rightarrow$ nuc synapses should decrease in strength when coactive with a subset of $g r \rightarrow P k j$ synapses that do not produce a decrease in Purkinje cell activity. This suggests that each $g r \rightarrow P k j$ synapse competes with its cohorts to make the strength of the $\mathrm{mf} \rightarrow$ nuc synapses consistent with the response that it encodes: $g r \rightarrow P k j$ synapses that have undergone LTD act to make the $\mathrm{mf} \rightarrow$ nuc synapses stronger, and synapses that have undergone LTP act to make $\mathrm{mf} \rightarrow$ nuc synapses weaker.

In this view, the amount of plasticity at $\mathrm{mf} \rightarrow$ nuc synapses could depend on the type of training. Standard delay conditioning, using a single CS and a fixed ISI, may represent an example where a limited number of $g r \rightarrow P k j$ subsets code for a CR and would lead to a moderate amount of plasticity at $\mathrm{mf} \rightarrow$ nuc synapses-enough to allow for the expression of small CRs following removal of the cerebellar cortex. In contrast, training with several auditory CSs, or training with a single CS using several ISIs, could contribute to more plas-

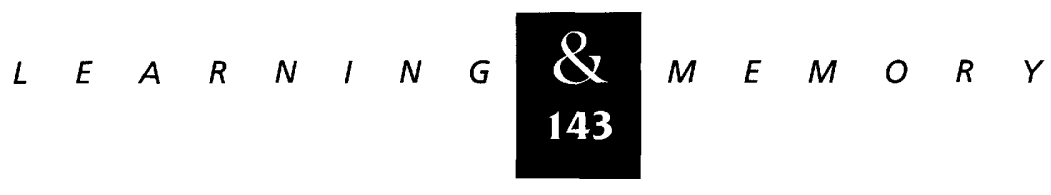


ticity in the nucleus because a CR is required under a larger number of circumstances-that is, more $g r \rightarrow P k j$ subsets code for a CR. Consequently, after a lesion of the cerebellar cortex, a single CS should elicit a relatively robust but still inappropriately timed CR.

Our assumptions also predict that the relative distribution of plasticity varies throughout extinction training and that the distribution of synaptic weights following acquisition and extinction may be quite different than before training. During extinction training, the previously learned decrease in Purkinje cell activity during the CS is reversed by the induction of LTP at CS-activated $g r \rightarrow P k j$ synapses. As with acquisition, our assumptions allow that CRs could be extinguished by the restoration of ongoing Purkinje activity during the CS, before a significant decrease in the strength of $\mathrm{mf} \rightarrow$ nuc synapses. Although further extinction training my also reverse the $\mathrm{LTP}_{\text {nuc }}$ at $\mathrm{mf} \rightarrow$ nuc synapses, the model predicts that a lesion of the cerebellar cortex might unmask CRs (albeit poorly timed) at the point in extinction when CRs initially fail to occur.

\section{SAVINGS}

The interactions we propose between two sites of plasticity offer possible explanations for two phenomena generally referred to as savings. One form of savings is the commonly observed finding that reacquisition following extinction occurs more rapidly than initial acquisition. Because our assumptions allow that a residual portion of the increased $\mathrm{mf} \rightarrow$ nuc synaptic strength produced by acquisition may persist following extinction training, subsequent reacquisition would occur relatively rapidly as LTD develops at $g r \rightarrow P k j$ synapses. At some level of abstraction, this reasoning is similar to the two-stage model of acquisition and extinction proposed by Kehoe (1988). However, unlike Kehoe's model, the present model predicts that with extended extinction training, the amount of savings should be reduced as $\mathrm{mf} \rightarrow$ nuc synapses decrease in strength as the result of repeated activation in mossy fiber collaterals at a time when the nucleus cell is inhibited.

This putative mechanism may also account in part for a second savings phenomenon in which training to one $\mathrm{CS}$ can facilitate acquisition of responses to a second CS. For this mechanism to apply, there must be a degree of overlap in the mossy fiber representations of the two CSs. Al- though there may be little to no behavioral generalization between the stimuli owing to virtually nonoverlapping granule cell representations, the potential for savings in acquisition to the second CS following training to the first should parallel the extent of the shared mossy fiber representation. This account predicts that following training to the first CS, a lesion of the cerebellar cortex should unmask a generalized response to the second CS. Although previous studies have shown that stimulus generalization increases somewhat following a cerebellar cortex lesion (Perrett et al. 1993; S. Garcia and M.D. Mauk, unpubl.), this increase may not be sufficient to explain all savings of this form. For example, it may not explain savings to a visual CS following training to an auditory CS. Our ideas also do not explain the savings seen with training to one eye following prior training to the other eye. An explanation of these observations appears to require additional mechanisms.

\section{RESPONSE TIMING AND THE ISI FUNCTION}

Our explanations for both the ISI function and for response timing arise almost entirely from assumptions about how the CS representation in the cerebellum changes during the stimulus. We have extended Marr's (1969) original idea-that stimuli are represented by the subsets of granule cells they make active-by suggesting that interactions between the populations of granule and Golgi cells activate different subsets of granule cells throughout a stimulus. Results from recent large-scale computer simulations of the cerebellar cortex demonstrate that, in principle, networks with an organization similar to the cerebellum produce time-varying representations from tonic, time-invariant mossy fiber inputs (Buonomano and Mauk 1994). These simulations also illustrated a possible explanation for the anticipatory nature of CRsthat is that responses begin before US onset. Because the cascade from one active subset of $g r \rightarrow P k j$ synapses to the next is continuous rather than discrete, the overlap between the currently active subset and the previously reinforced subset will increase gradually as the reinforced time approaches. As the similarity increases, Purkinje cell activity will decrease, and thus the likelihood of a response will grow, producing an anticipatory CR.

The time-varying representation of the CS generated by the cerebellar cortex may come at a cost: a sensitivity to noise that gradually degrades the

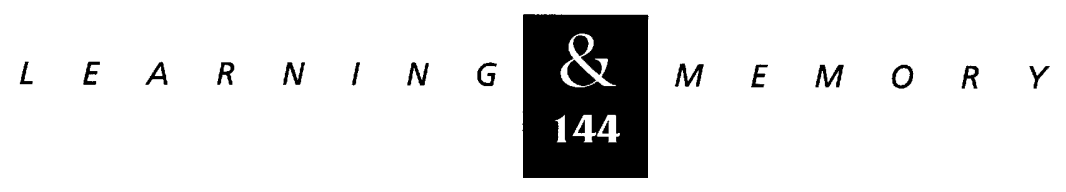


across-trials consistency of granule cell activity as the CS duration increases. As we noted previously, the ISI function may arise from a rapid increase then gradual decline in the consistency from one trial to the next in the subsets of granule cells activated by the CS.

The present ideas point to the importance of characterizing the CS-related firing patterns of granule cells over many trials. Unfortunately, granule cells are small and densely packed, which makes unit recordings from awake animals extremely difficult. We know of no published unit recordings of cerebellar granule cells during Pavlovian conditioning or even during presentation of CS-like stimuli. Given the statistical and distributed nature of the CS representation we propose, empirical tests would require recording single unit responses to a large number of CS presentations from a large number of granule cells. However, if such recordings could be obtained, the peristimulus histograms should show peaks and/or valleys during the CS and different cells should have these peaks and valleys in different times. This distribution would provide the temporal discrimination for response timing. Furthermore, the overall likelihood of observing these peaks and valleys during segments of the CS, in each of a large number of cells, should be related to across-trials consistency and thus should parallel the time course of the ISI function.

A number of neural-like models that involve time-varying $\mathrm{CS}$ representations have been proposed to account for the timing of CRs. For example, Moore and colleagues (1986) presented a tapped delay line model in which the CS activates a serial sequence of neurons, with each synapse producing a delay. These delay lines, combined with an eligibility period for plasticity, provide an account for response timing and for the ISI function (Moore et al. 1986, 1989; Moore and Blazis 1989). Bullock and colleagues (1994) presented a cerebellar model adapted from a timing hypothesis originally proposed to occur in the hippocampus (Grossberg and Schmajuk 1989) in which response timing is derived from an array of CS-activated elements with different time constants. In a similar fashion, Gluck and colleagues (1990) proposed a time-varying CS representation comprised of CS elements that oscillate at different frequencies and phases.

Clearly, there are many ways in which neural activity related to a CS could vary over time. When combined with an activity-dependent form of syn- aptic plasticity, such a CS representation could generate CRs with the appropriate timing. However, the key question is how the nervous system generates such a time-varying representation. Rather than building in time-varying activity with assumptions about delay-lines or about arrays of cells or units with different temporal properties (membrane or synaptic time constants, oscillations at different frequencies, etc.), our approach has been to ask if time-varying activity can emerge naturally from networks configured like the cerebellar cortex (see Buonomano and Mauk 1994).

We have used the same approach in attempting to account for the ISI function. We have asked whether, like CR timing, a mechanism for the ISI function can arise as a network property of the anatomy and physiology of the cerebellar cortex. This is in contrast to previous models in which the ISI function has generally been obtained by building in an eligibility parameter for plasticity that explicitly mirrors or reproduces the time course of the ISI function (Grossberg and Schmajuk 1989; Moore and Blazis 1989; Moore et al. 1989). Our account for the ISI function is obtained without the addition of noncerebellar features and without imposing parameters explicitly calculated to produce the ISI function. This mechanism also permits eligibility for plasticity to be related to the ongoing CS-related spike activity (in the $g r \rightarrow P k j$ synapses). For example, climbing fiber inputs can simply modify the strength of those $g r \rightarrow P k j$ synapses that are active. There is no need to assume that climbing fiber inputs interact with an eligibility signal whose time course explicitly mirrors and directly produces the ISI function, as is the case with many models.

\section{PERMANENT LESIONS OF THE CEREBELLAR CORTEX}

Because the cerebellar nucleus is assumed to be necessary for the expression of CRs, the ability of the model to explain findings that interpositus nucleus lesions abolish CRs is unsurprising. On the other hand, predictions regarding the effects of cerebellar cortex and IO lesions are derived from the interactions of several assumptions of the model.

A firm prediction of the model is that the timing of any CRs observed following lesions of the cerebellar cortex should be disrupted in a characteristic manner. Postlesion responses should show

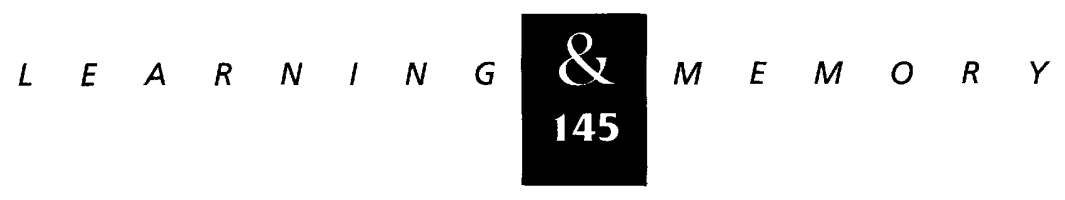


short latencies to onset and to peak that are, at most, minimally sensitive to the ISI used in training (that is, to the extent that the strength of the $\mathrm{mf} \rightarrow$ nuc synapses can influence the time required to reach threshold for response production). These expectations have been confirmed by Perrett et al. (1993). This study used within-subject comparisons in which animals were trained with two CSs, each reinforced at a different ISI. This training produced differently timed responses to the two CSs. Following lesions of cerebellar cortex, latencies to onset and to peak were short, and the differential timing observed prior to the lesion was abolished; i.e., the postlesion CRs displayed the same, short latency independent of their prelesion timing. These findings are consistent with the proposal that under most circumstances, CRs in a well-trained animal are mediated by plasticity in both the cerebellar cortex and nucleus. Disruption of CR timing is consistent with the assumption that the cerebellar cortex generates a time-varying representation of the CS in the cortex and that $g r \rightarrow P k j$ synapses active at the time of US occurrence undergo plasticity. Retention of improperly timed CRs following cortex lesions is consistent with the idea that plasticity also occurs at the $\mathrm{mf} \rightarrow$ nuc synapses.

In the previous sections we outlined reasons why the retention of CRs following lesions of the cerebellar cortex should depend in complex ways on the amount and type of prelesion training. This leaves open the possibility that cerebellar cortex lesions will abolish responses early in training and will spare improperly timed responses after additional CS + US training. We believe that this possibility may allow a better understanding of apparent inconsistencies in the effects of cerebellar cortex lesions on the retention of CRs that have been reported. The original report on the effects of cerebellar cortex lesions (McCormick and Thompson 1984), as well as many other subsequent studies (Perrett et al. 1993; Perrett and Mauk 1995; L.S. Garcia and M.D. Mauk, unpubl.), shows retention of CRs following cerebellar cortex lesions. However, in other studies (Yeo et al. 1985b; Yeo and Hardiman 1992), similar lesions abolished the retention of previously acquired eyelid responses. As has been argued by Lavond and Steinmetz (1989), one explanation of these differences suggests that the abolished responses reflect unintended damage to the underlying interpositus nucleus that cannot be detected by histological analysis. Although this is possible, our model allows a different per- spective that is amenable to experimental evaluation-that differences in the amount and type of training that an animal has had before the lesion can explain the variable lesion effects that have been reported. For example, with modest amounts of training, CRs may be mediated largely by LTD in the cortex, whereas with extended training, CRs may be produced by plasticity in both the cerebellar cortex and interpositus nucleus. The effects of a cerebellar cortex lesion on the retention of CRs would be quite different for these two circumstances (Mauk 1997).

Another strong prediction of the model is that cerebellar cortex lesions should prevent both the extinction of previously learned responses and the acquisition of new CRs. Although, within our assumptions, one site of plasticity ( $\mathrm{mf} \rightarrow$ nuc synapses) is spared by such a lesion, these predictions arise from the idea that plasticity at this remaining site requires either Purkinje cell input (for LTD $_{\text {nuc }}$ ) or transient release from Purkinje cell input (for LTP $_{\text {nuc }}$ ). If this is true, the amplitude of the response seen initially after the lesion should not change with further acquisition or extinction training. A recent experiment has shown that lesions of the cerebellar cortex prevent extinction of eyelid CRs over extensive CS-alone training (Perrett and Mauk 1995). This study and a previous study (Perrett et al. 1993) found that lesions must include the anterior lobe of the cerebellar cortex to affect both the timing of the CRs and extinction. In addition, preliminary results of Garcia and Mauk (1995) also support the prediction that cerebellar cortex lesions prevent acquisition of new responses. This study also confirmed that damage to the anterior lobe is required to produce the effect. Together, these studies are consistent with the hypotheses that (1) CRs in well-trained animals are mediated by plasticity in both the cerebellar cortex and nucleus, (2) both a time-varying CS representation and synaptic plasticity in the cerebellar cortex are responsible for CR timing, and (3) following lesions of the cerebellar cortex, the ability of $\mathrm{mf} \rightarrow$ nuc synapses to undergo plasticity is greatly diminished.

In contrast, two previous studies showed that cerebellar cortex lesions produce deficits in, but do not completely abolish, acquisition (Lavond et al. 1987; Lavond and Steinmetz 1989). The histological reconstructions presented in these papers indicate that considerable parts of the anterior lobe were spared in these experiments. We believe that an appropriate test must focus on the involvement

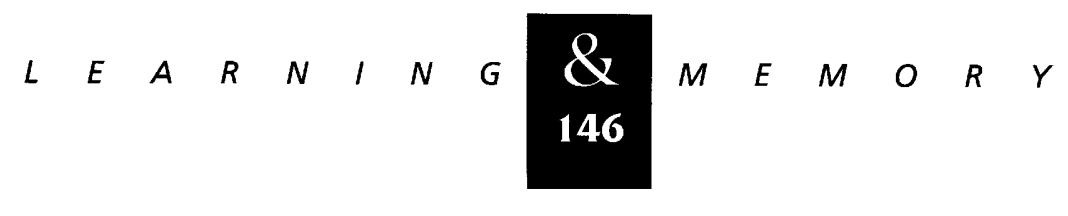


of the anterior lobe and at the same time be very careful to provide assurances that there is no damage to structures known to be necessary for the expression of responses such as the interpositus nucleus or the middle or superior cerebellar peduncles.

\section{GENETIC MANIPULATIONS OF CEREBELLAR CORTEX}

Recently, mice with genetic mutations that affect the cerebellum have been used to address cerebellar involvement in eyelid conditioning. In one such study, Chen et al. (1996) report that $p c d$ mice, which lose all Purkinje cells in the third to fourth week of development (functionally disconnecting the cerebellar cortex from the cerebellar nuclei), show some acquisition of eyelid CRs, although acquisition is "impaired profoundly" (p. 2834). The authors also report that these CRs extinguish over 4 days of CS-alone presentations. The authors conclude that "This finding suggests that the Purkinje cells and thus the entire cerebellar cortex (because the Purkinje cells are the sole efferent neurons of the cortex) are not necessary for the extinction of eyeblink CRs" (p. 2835). Another study, using mice lacking one isoform of metabotropic glutamate receptors $\left(\mathrm{mGluR}_{1}\right)$, used in vitro cerebellar slice to show a lack of LTD at $g r \rightarrow P k j$ synapses. This deficit in LTD was accompanied by an impairment, but not complete abolition, of the ability to acquire conditioned eyelid responses (Aiba et al. 1994; see also Chen et al. 1995).

Although these results appear to contradict previous lesion results and contradict predictions of the present model, their implications for cerebellar mechanisms of eyelid conditioning are not yet entirely clear owing to a number of unresolved issues that make drawing conclusions from the findings difficult. For example, Chen et al. (1996) acknowledge a number of ways in which the $p c d$ mutant mice differ from wild type. In addition to lacking Purkinje cells, they have smaller IO cell bodies, show abnormal neurochemistry in the interpositus nucleus (Triarhou and Ghetti 1991; Goldowitz and Eisenman 1992; Godlett et al. 1992), show ataxia, and fail to learn the hidden platform problem in the Morris water maze (suggesting abnormal development of the hippocampus). These specific abnormalities exemplify the major conceptual problems with most learning studies using mutant mice, namely, the potential for widespread abnormalities (both obvious and subtle) and the corresponding potential for compensatory mechanisms to be engaged during development or during learning. Thus, to conclude that learning abilities observed in mutant animals demonstrate that the most obviously affected brain systems are not involved in such learning in normal animals is problematic. To make such a claim, given the potential for the many confounds described above, it is absolutely essential to show that any residual learning (or extinction) in mutant animals is mediated by the same processes involved in normal animals.

Taking the Chen et al. (1996) observation of extinction in mice with no Purkinje cells as an example, we note first the absence of controls critical for determining whether the loss of responding during 4 days of CS-alone training was attributable to extinction rather than forgetting. Given the variety of structural and physiological abnormalities in these mice, there is no way of knowing whether or not retention is normal or whether forgetting is more rapid than in wild-type mice. Second, if CRs do indeed extinguish in these animals, it is important to know whether the extinction is mediated through a compensatory mechanism or the mechanism that operates in normal mice. It is also important to determine whether the impaired, but still present, acquisition seen in the mutant mice depends on the cerebellum. Unfortunately, the key question of whether lesions of the interpositus nucleus in the mutant mice abolish the small CRs that are acquired was not evaluated. Without such controls, it seems quite possible that the (impaired) acquisition that is seen in mutant mice may be attributable to compensatory, even noncerebellar, mechanisms.

\section{PERMANENT LESIONS OF THE IO}

The assumptions of the model do not permit a firm prediction concerning the retention of CRs following a lesion of the IO. The uncertainty stems from the unknown effects of removing spontaneous climbing fiber activity. Without it, $g r \rightarrow P k j$ synapses would undergo LTP each time they are active, and, presumably, this would lead to a reduction or abolition of previously trained CRs. The surest way of activating these synapses is to present the CS. However, they will also be active to a lesser degree at other times, and in both cases this should induce LTP and diminish the probability of

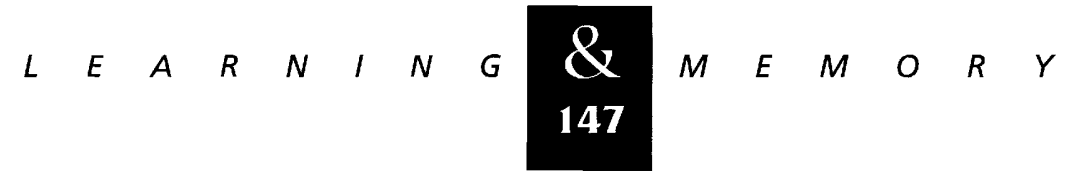


CRs. Thus, the interval between the IO lesion and testing for retention of CRs could affect the ability of the CS to elicit a CR. Relatively long lesion-to-test intervals should reduce the likelihood of observing postlesion CRs, as should recovery in environments that increase the probability that the $g r \rightarrow P k j$ synapses coding the pretrained CS are active during the recovery period (e.g., a noisy home case environment). Various combinations of these factors could be responsible for the differences in the results of two studies examining IO lesions, one of which reported an abolition of CRs (Yeo et al. 1986) and the other of which reported initial retention of CRs (McCormick et al. 1985). In addition, the lesions reported by Yeo et al. were relatively large compared to those reported by McCormick et al. and may therefore have abolished CRs as a result of more widespread disruption of cerebellar function. However, our model makes the firm prediction that any responses retained after an IO lesion will extinguish with additional CS + US training, which was observed in the McCormick et al. study.

\section{REVERSIBLE LESIONS OF THE INTERPOSITUS NUCLEUS AND IO}

Several studies have used reversible lesions using local anesthetics such as lidocaine or specific transmitter agonists or antagonists to analyze cerebellar involvement in eyelid conditioning. Mamounas et al. (1987) reported that infusion of the $\mathrm{GABA}_{\mathrm{A}}$ antagonists into the interpositus nucleus abolishes CRs for 10-20 trials, followed by a gradual return to preinfusion baseline. Because at least some of the animals received extensive preinfusion training, our assumptions predict significant plasticity at $\mathrm{mf} \rightarrow$ nuc synapses, and thus we cannot explain the initial abolition of CRs. To the degree that the drug effect simulated removal of the cerebellar cortex, we would expect that response timing during the recovery phase would be disrupted. Unfortunately, response timing was not investigated in this study.

In an elegant and important series of studies, Welsh and Harvey (1991) and Krupa et al. (1993) assessed the effects of inactivating the interpositus nucleus with lidocaine during acquisition training. Welsh and Harvey observed significant retention of CRs in post-training tests during which the interpositus nucleus was free of lidocaine. Using a similar protocol, Krupa et al. found no retention of responses following a training phase during which the nucleus cells were hyperpolarized with the GABA agonist muscimol.

We suggest that both observations are consistent with the present model and that the different modes of action of lidocaine and muscimol led to the different experimental outcomes. We believe that the induction of LTD at $g r \rightarrow P k j$ synapses and the acquired decreases in Purkinje activity proceeded normally in both experiments, perhaps even more robustly than normal without responserelated inhibition of climbing fibers. However, because lidocaine blocks both pre- and postsynaptic action potentials, no activity-dependent plasticity should occur in the interpositus nucleus during its infusion. The subsequent expression of CRs in the absence of lidocaine would reflect training-induced plasticity in the cerebellar cortex sufficient to generate CRs, even though the strength of nucleus synapses remained unchanged. In contrast, our assumptions predict that training during infusion of muscimol in the interpositus would promote extinction-like processes at the $\mathrm{mf} \rightarrow$ nuc synapses, that is, a decrease in synaptic strength owing to activation of $\mathrm{mf} \rightarrow$ nuc synapses during conditions that mimic strong Purkinje cell input (i.e., muscimol). Without knowing how this mix of functionally opposing synaptic changes in the cortex and nucleus would affect cerebellar output, it is difficult to predict the degree of retention that would be seen. Overall, our assumptions are qualitatively consistent with the immediate appearance of CRs after training using an infusion of lidocaine and with a more delayed appearance of CRs observed following training with muscimol infused into the interpositus nucleus.

More recently, Nordholm et al. (1993) demonstrated that infusing lidocaine into dorsal regions of interpositus nucleus prevents acquisition of CRs, whereas infusing it into more ventral regions does not (even though it still blocks the expression of CRs). At face value, these data contradict the hypothesis that conditioning is driven by training-induced plasticity in the cerebellar cortex and, as such, would require a major modification of the present model. However, one important possibility that was not excluded in these experiments is that lidocaine infused into the dorsal regions blocked climbing fiber or mossy fiber inputs to the cerebellar cortex.

Finally, one study has shown that cooling the IO produces increases in Purkinje cell activity that reverse with the termination of cooling (Montarolo

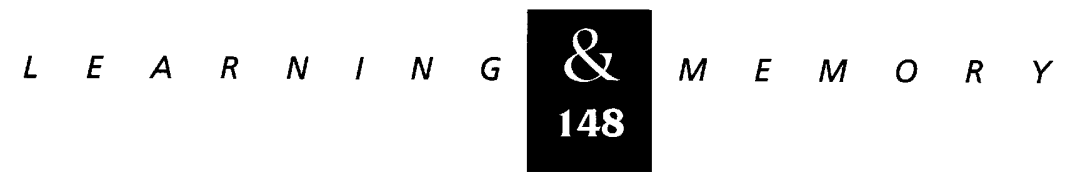


et al. 1982). These results are consistent with the proposed self-regulating modulation of climbing fiber activity. Without appropriate levels of spontaneous climbing fiber activity (e.g., during cooling), the induction of LTP at $g r \rightarrow P k j$ synapses, unopposed by LTD, would lead to increased activity of Purkinje cells.

\section{PREDICTIONS OF THE MODEL RELATED TO REVERSIBLE LESIONS}

In general, the use of reversible lesions is fertile ground for further investigating cerebellar involvement in motor learning such as eyelid conditioning. One important aspect of our model is the large number of empirically testable predictions that it makes regarding the effects of various reversible lesion experiments.

Our assumptions suggest that the extinction of CRs should be blocked by any reversible lesion that disrupts the inhibitory pathway from cerebellar nuclei to the climbing fibers in the IO. Under such circumstances, the presentation of CS-alone extinction trials would not be expected to induce LTP at the CS-activated $g r \rightarrow P k j$ synapses because the response-related inhibitory output from the cerebellum to the IO is required to reduce spontaneous climbing fiber activity below the equilibrium level. According to this thinking, extinction should not occur when the CS-alone extinction trials are delivered during the following reversible lesions: (1) any infusion that reversibly blocks the ability of interpositus nucleus output to inhibit climbing fiber activity, such as lidocaine or muscimol in the interpositus nucleus or lidocaine in the superior cerebellar peduncle and (2) infusion of GABA antagonists into the IO, which should also disrupt inhibition of climbing fibers initiated by cerebellar output.

In addition, the model predicts that infusions that block extinction should also abolish the shift in response timing seen when training begins with one ISI and then is shifted to a second ISI. For example, Coleman and Gormezano (1971) and also Millenson et al. (1977) initially trained animals at one ISI until robust CRs were observed; additional training was then conducted at a different ISI. The authors showed that changing the ISI produced a gradual shift in response timing; latencies to onset and to peak gradually became appropriate for the second ISI. Given our assumptions, the timing shift seen in this protocol involves the extinction of the response timed appropriately for the first ISI and the simultaneous acquisition of a response with timing appropriate for the second ISI. With extinction disrupted, shifting to the new ISI should result in acquisition of a response timed appropriately for the second ISI without extinguishing the first, producing double-peaked responses as has been observed by D.J. Krupa, J. Weng, and R.F. Thompson (unpubl.).

It is also clear from the assumptions of the model that reversible lesions that prevent activity in Purkinje cells or disrupt their inhibitory input to nucleus cells should also disrupt the timing of CRs. Moreover, to the extent that rebound excitation contributes to activation of nucleus cells, such manipulations may decrease the amplitude of CRs as well. These effects should be produced by the following reversible lesion protocols: (1) GABA antagonists are infused into the interpositus nucleus to block the inhibition of nucleus cells by Purkinje cells. Unless these antagonist infusions involve complex interactions with local inhibitory interneurons, the effects of such infusions should be the equivalent of a reversible disconnection of the cerebellar cortex from the nucleus. (2) Local anesthetics or the GABA agonist muscimol is infused into the anterior lobe of the cerebellar cortex. With no inhibitory input, the nucleus cells could become active as soon as the $\mathrm{CS}$ input arrives via the mossy fibers. This protocol should reduce the amplitude of the CRs by removing the contribution of the cortex to CR generation (i.e., by removing rebound excitation in the nucleus cells).

In addition, the infusion of a GABA antagonist such as picrotoxin into the anterior lobe of the cerebellar cortex should abolish the expression of CRs. Without inhibitory input from stellate and basket cells, the decrease in excitatory synaptic input that occurs during the CS owing to training and the induction of LTD may not be sufficient to produce a robust decrease in the activity of the Purkinje cell. If so, the ongoing Purkinje cell inhibition of nucleus cells should prevent the expression of the already learned responses.

\section{OTHER CONDITIONING PHENOMENA}

Our assumptions also provide fairly simple accounts for more complex aspects of conditioning such as blocking, conditioned inhibition, differential conditioning, and trace conditioning. The Kamin blocking effect is the finding that the amount of conditioning produced by a CS + US pairing is diminished when the target CS appears in com-

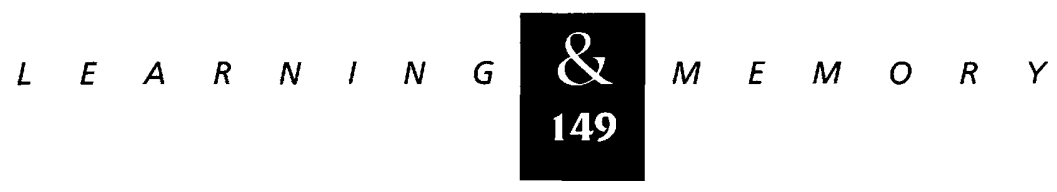


pound with a second CS that has previously been trained with that US, compared with occasions in which the target is trained in compound with a second CS that has not been paired with the US (Kamin 1969). A well-controlled design would look like the following:

\begin{tabular}{cccc}
\hline & Phase 1 & Phase 2 & Test \\
\hline Blocking & CS1 $\rightarrow$ US & $(\mathrm{CS} 1+\mathrm{CS} 2)$ & responding \\
& & $\rightarrow$ US & to CS2 \\
Control & CS3 $\rightarrow$ US & $(\mathrm{CS} 1+\mathrm{CS} 2)$ & \\
& & $\rightarrow$ US & \\
\hline
\end{tabular}

The blocking effect is the finding that during the test phase, the level of conditioned responding to CS2 is lower in the blocking group than in the control group. Thus, prior training to CS1 blocked conditioning to CS2. The phenomenon of blocking is quite important because it demonstrates clearly that although the pairing of a CS and US is necessary for conditioning to occur, it is not a sufficient condition. Why this should be so is a question that has motivated a considerable amount of experimental and theoretical work in the Pavlovian conditioning literature (Rescorla and Wagner 1972; Makintosh 1975; Wagner 1976, 1979, 1981; Pearce and Hall 1980).

Successful theoretical accounts for blocking have been obtained in a number of models that assume that an expected US is less effective than an unexpected US (e.g., Wagner and Rescorla 1972; Wagner 1976, 1981; Sutton and Barto 1981). Moreover, a number of authors have noted that the inhibition of climbing fibers by cerebellar output represents a potential biological implementation of this theoretical construct (e.g., Donegan et al. 1989). An account of blocking in the present model is obtained in this fashion and is presented only to highlight aspects that may be unique to our model. Essentially, the prior training to CS1 in the blocking group produces a non-zero level of responding to the CS1 + CS2 compound in phase two. As such, less learning occurs during the compound because the US-evoked climbing fiber response is somewhat diminished, resulting in less conditioning to the CS2.

A number of experimentally testable predictions arise from the assumption that blocking is produced by increased inhibition of climbing fibers during the compound phase. The blocking effect should be sensitive to the reversible lesions that prevent cerebellar output from inhibiting climbing fiber activity during the second phase. During the phase 2 compound training, lidocaine or muscimol infused in the cerebellar nucleus or a GABA antagonist such as picrotoxin infused in the IO should prevent blocking. These predictions are consistent with preliminary findings of J.J. Kim, D.J. Krupa, and R.F. Thompson (unpubl.) who report that intra-olivary infusions of picrotoxin prevent blocking in rabbit eyelid conditioning. Moreover, given mechanisms of temporal discrimination in the cerebellar cortex that we hypothesize, the present model makes the prediction that blocking may show a degree of dependency on the ISI. Specifically, if the ISI in phase 1 is not the same as in phase 2, then blocking should be attenuated. This prediction simply acknowledges the expectation that the CR-related inhibition of climbing fibers should show ISI-dependent timing similar to the CRs themselves. To our knowledge, this prediction has not yet been tested experimentally.

An alternative way in which the effectiveness of the US might be reduced could be through the desensitization of the trigeminal nerve produced by the closure of the eyelid during the CR (Pellegrini and Evinger 1995). Although such effects may contribute to the blocking effect, we would predict that if the facial motor nucleus was inactivated by lidocaine during the compound phase of the training phase (thereby preventing CS1 from eliciting a CR), a substantial amount of blocking would be observed in the test phase in the absence of lidocaine. That is, responding to CS2 would still be significantly lower in such a blocking group, compared with the control group.

Our assumptions suggest a parallel account for conditioned inhibition, a phenomenon that was first reported by Pavlov (1927) and is procedurally quite similar to blocking. The main difference is that the compound (CS1 + CS2) is not reinforced in the second phase. An example of a procedure for developing and demonstrating conditioned inhibition is

\begin{tabular}{|c|c|c|c|}
\hline & Phase 1 & Phase 2 & Test \\
\hline \multirow{2}{*}{$\begin{array}{c}\text { Conditioned } \\
\text { inhibition }\end{array}$} & $\mathrm{CS} 1 \rightarrow \mathrm{US}$ & $(\mathrm{CS} 1+\mathrm{CS} 2)$ & \\
\hline & $\mathrm{CS} 3 \rightarrow \mathrm{US}$ & $\rightarrow$ no US & \\
\hline \multirow[t]{2}{*}{ Control 1} & $\mathrm{CS} 3 \rightarrow \mathrm{US}$ & $(\mathrm{CS} 1+\mathrm{CS} 2)$ & CS3 alone vs. \\
\hline & & $\rightarrow$ no US & $(\mathrm{CS} 2+\mathrm{CS} 3)$ \\
\hline \multirow[t]{2}{*}{ Control 2} & $\mathrm{CS} 1 \rightarrow \mathrm{US}$ & $\mathrm{CS} 1 \rightarrow$ no US & \\
\hline & CS3 $\rightarrow$ US & $\mathrm{CS} 2 \rightarrow$ no US & \\
\hline
\end{tabular}

$$
\begin{array}{lllllllllllllll}
\mathcal{Q} & E & A & R & N & I & N & G & M & E & M & O & R & Y \\
150 & & & & & &
\end{array}
$$


In the above procedure, conditioned inhibition would be revealed as a significantly greater ability of CS2 to diminish responding to CS3 in the conditioned inhibition group compared with the control groups. Among other things, the control groups allow one to evaluate the nonassociative, disruptive effects of $\mathrm{CS} 2$ on responding to CS3, for example, external inhibition (Pavlov 1927; Boakes and Halliday 1972; Wagner and Rescorla 1972).

The model assumes that CS2 develops inhibitory capacities during phase 2 as a result of CS1 decreasing the spontaneous climbing fiber activity below equilibrium, allowing $g r \rightarrow P k j$ synapses to undergo LTP. Consequently, $g r \rightarrow P k j$ synapses coding CS2 will have an increased excitatory drive on the Purkinje cells, which will result in an increased inhibitory drive onto the interpositus nucleus, making it more difficult for excitatory CSs to activate the output cells and initiate a CR. The model predicts that disrupting the inhibitory pathway from the cerebellum to the climbing fibers (during phase 2) will prevent conditioned inhibition and that conditioned inhibition should show temporal specificity.

\section{DISCRIMINATION TRAINING}

The above conceptualization also provides a way of understanding several observations related to differential conditioning, which involves separate presentation of two CSs, one of which is reinforced (designated CS+) and one of which is not (CS-). The degree of differential conditioning is defined as the difference in responding between the CS+ and the CS-. To the extent that there is generalization between the two CSs, responses are elicited by both CSs early in training. However, with additional training the responses to the CS+ continue to increase and the responses to the CSdecrease (e.g., Moore 1972).

During initial stages of training, as both CSs acquire the ability to elicit responses-the CS+ directly and the CS- through the activation of common $g r \rightarrow P k j$ synapses-each CS would then begin to inhibit climbing fiber activity. On a CS- trial, inhibition of climbing fiber activity would act to increase the strength of the $g r \rightarrow P k j$ synapses activated by the CS-, reduce the probability of a CR, and thereby increase the differential responding between the CS+ and the CS-. Thus, differential conditioning would be the result of training-induced LTD in the $g r \rightarrow P k j$ synapses activated by the CS+ and training-induced LTP in the $g r \rightarrow P k j$ synapses activated by the CS-. Moreover, the induction of LTP at these synapses would occur only after the CS- had initially acquired the ability, through generalization, to activate the interpositus output cells and depress climbing fiber activity.

The model predicts that the synapses unique to the CS+ would undergo more LTD than normal and that synapses unique to the CS- would undergo LTP, preventing responding to the CS- (cf. Blough 1975; Rescorla 1976). Synapses common to both CSs would initially undergo LTD (which would be responsible for responding to CS- early in training) but would then undergo LTP so that eventually they would show a relatively smaller net change. Because increasing the discrimination ratio (the difference in responding between the CS+ and the CS-) when there is substantial generalization early in training requires that elements unique to the CS- must become conditioned inhibitors, we predict that differential conditioning would be disrupted by any of the above manipulations assumed to prevent conditioned inhibition or extinction. We predict that such manipulations would increase responding to both CSs and that responding to CS- would not decrease with continued training.

\section{TRACE CONDITIONING}

The model can provide an account for trace conditioning with the assumption that the timevarying pattern of granule cell activity can persist for a period of time following CS offset. In this case, the decline in conditioning seen as the trace interval increases would largely reflect a decrease in CS-related activation of granule cells and a return to background levels of granule cell activity. However, since Moyer et al. (1990) found that hippocampal lesions prevent trace conditioning with long trace intervals, another possibility is that the hippocampal formation can provide inputs to mossy fibers during the trace interval. This interpretation is consistent with observations that both cerebellar and hippocampal lesions block trace conditioning.

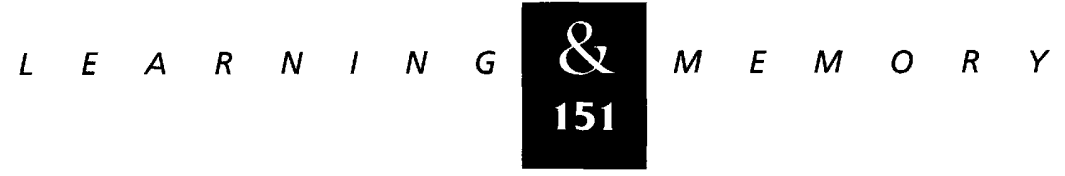


THE RELATIONSHIP BETWEEN LTD AND EYELID CONDITIONING

Certain features of LTD and of the cerebellarolivary system have raised concerns over the likelihood that LTD, or any form of climbing fiberinduced plasticity in the cerebellum, is involved in motor adaptation. These have included the following four points: (1) Cerebellar cortex lesions don't always abolish CRs (McCormick and Thompson 1984; Yeo and Hardiman 1992; Perrett et al. 1993; Perrett and Mauk 1995), (2) the timing required of granule cell and climbing fiber inputs to induce LTD does not correspond to the intervals between CS and US that promote acquisition of eyelid responses (Schreurs and Alkon 1993, 1996; Chen and Thompson 1995), (3) the number of trials required to acquire eyelid responses is much larger than the number of granule cell-climbing fiber pairings required to induce LTD, and (4) climbing fibers are spontaneously active (Keating and Thach 1995).

We are encouraged that each of these apparent problems are obviated within the context of our assumptions. The lesion data can be explained by assuming that (1) cerebellar cortex lesions remove one site of plasticity and remove an input that is critical for the induction of plasticity at the second site and that (2) the relative contributions of plasticity in the cerebellar cortex and nuclei can depend on the amount and type of training. Thus, the predictions of the present model are consistent with the observations from both eyelid conditioning and from VOR adaptation that cerebellar cortex lesions prevent acquisition and extinction of responses and yet allow variable degrees of retention of previously learned responses (see Robinson 1976; Raymond et al. 1996; Mauk 1997).

The lack of correspondence between both (1) the timing required for LTD and the ISIs that support conditioning and (2) the trials required to induce LTD versus the trials required to acquire eyelid responses can be explained by the time-varying granule cell representation of the CS. To promote the acquisition of properly timed CRs, climbing fiber inputs can, as is observed, modify only those $g r \rightarrow P k j$ synapses that are active during that segment of the CS. Moreover, a lack of perfect consistency in the CS representation from one trial to the next can explain why a large number of trials are required for $\mathrm{CR}$ acquisition, whereas a much smaller number of pairings are required to induce LTD. Finally, the ideas we present suggest a positive role for spontaneous activity, rather than the potential deleterious effects that have been suggested. Properly regulated spontaneous climbing fiber activity could help maintain the pattern of synaptic strengths encoding the memories for previous motor learning, as suggested by Keating and Thach (1995).

\section{Conclusions}

As stated in the introduction, our purpose in presenting this model was to describe structural and functional properties of the cerebellum that could account for the corpus of eyelid conditioning data. Our intent was also to use the model to generate experimentally testable predictions and to point to new experiments that may facilitate a better understanding of the neural basis of eyelid conditioning. We are encouraged by the breadth of data for which our assumptions suggest plausible accounts and by the recent studies that appear to confirm several predictions of the model. Mathematical analyses and biologically detailed computer simulations have also recently produced results consistent with the more complex properties of the cerebellum that are suggested by our model (Buonomano and Mauk 1994; G.T. Kenyon, J.F. Medina, and M.D. Mauk, unpubl.). However, many of the predictions of the model have not yet been tested, and thus its real value will be determined by the productivity of the experimental programs to which it points.

In the face of numerous studies implicating possible plasticity in other brain regions such as the hippocampus, our focus on cerebellar mechanisms of eyelid conditioning deserves some comment. This focus stems from our conviction that the existing data firmly indicate that the cerebellum is responsible for the expression of conditioned eyelid (and other skeletal muscle) responses. However, this does not in any way deny the involvement of, or plasticity in, other brain regions during Pavlovian eyelid conditioning. Experiments may be designed carefully to isolate and test a particular hypothesis, but the animals nonetheless learn many things when placed in conditioning chambers and presented with various stimuli. Given this fact, the evidence that cells in many regions seem to encode aspects of the conditioning regimen and that synapses in many regions appear to undergo plasticity that is conditioning related should not be surprising. The challenge is to understand the roles of this activity/plasticity and

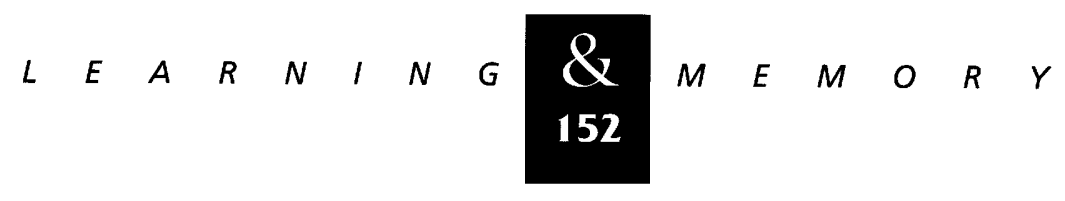


to avoid the overly simplistic view that the animal only learns what we measure. Thus, when the question is restricted to how the animal blinks when the tone is presented, we are convinced by the evidence that a strong case can be made that cerebellar mechanisms contribute to the acquisition and expression of CRs. We take the ability of the present model to provide plausible accounts for a wide range of eyelid conditioning phenomena as additional supporting evidence, for it indicates that networks with cerebellar-like properties can, in principle, generate a wide variety of phenomena observed in Pavlovian eyelid conditioning. We also propose that predictions of the model represent additional and fairly specific tests of cerebellar involvement in motor learning.

We fully acknowledge that a variety of Pavlovian phenomena fall outside the scope of our model. For example, the model obviously does not address conditioning phenomena thought to be dependent on the hippocampal formation: latent inhibition (Siegel 1972; Lubow 1973; Solomon and Moore 1975; Schmajuk and DiCarlo 1992; Schmajuk et al. 1996) and control of responding by configural cues (Whitlow and Wagner 1972; Sutherland et al. 1989; Jarrard and Davidson 1991; Rudy and Sutherland 1995). It has also been claimed that blocking is at least in part hippocampally dependent (e.g., Solomon 1977). The degree to which the blocking effect is the product of the hippocampal formation or the modulation of climbing fiber activity by inhibitory projections of the cerebellum to the IO remains to be determined. Clearly, the more general challenge is to specify how the hippocampal formation interacts with the cerebellar and brain-stem system we have outlined to generate the various eyeblink conditioning phenomena that are dependent on the hippocampal formation.

For now, the key aspects of this model are (1) its ability to explain acquisition and extinction and the apparent distribution of plasticity between cortex and nucleus suggested by lesion studies, (2) its ability to explain both response timing and the ISI function without hypothetical parameters that are specifically related to the ISI (such as arrays of time constants or time-varying eligibility periods for plasticity), (3) its ability to explain apparently contradictory results of reversible lesions of the interpositus nucleus, and most importantly, (4) the many specific and empirically testable predictions suggested by our assumptions.

\section{Acknowledgments}

We thank N. Waxham, D.V. Buonomano, S.P. Perrett, G.T. Kenyon, K.S. Garcia, P.M. Steele, and J.F. Medina for useful comments. Preparation of this manuscript was supported by Scholars' awards from the National Down Syndrome Society, the McKnight Foundation and grant MH46904 (M.D.M.), and the Whitehall Foundation (D90-30) (N.H.D).

The publication costs of this article were defrayed in part by payment of page charges. This article must therefore be hereby marked "advertisement" in accordance with 18 USC section 1734 solely to indicate this fact.

\section{References}

Aiba, A., M. Kano, C. Chen, M.E. Stanton, G.D. Fox, H. Herrup, T.A. Zwingman, and S. Tonegawa. 1994. Deficient cerebellar long-term potentiation and impaired motor learning in mGluR1 mutant mice. Cell 79: 377-388.

Aitkin, L.M. and J. Boyd. 1978. Acoustic input to the lateral pontine nuclei. Hear. Res. 1: 67--77.

Albus, J.S. 1971. A theory of cerebellar function. Math. Biosci. 10: 25-61.

Anderson, G., M. Garwicz, and G. Hesslow. 1988. Evidence for GABA-mediated cerebellar inhibition of the inferior olive in the cat. Brain Res. 472: 450-456.

Angaut, P. and C. Sotelo. 1989. Synaptology of the cerebello-olivary pathway. Double labeling with anterograde axonal tracing and GABA immunocytochemistry in the rat. Brain Res. 479: 361-365.

Artola, A., S. Bröcher, and W. Singer. 1990. Different voltage-dependent thresholds for inducing long-term depression and long-term potentiation in slices of rat visual cortex. Nature 347: 69-72.

Blough, D.S. 1975. Steady state data and quantitative model of operant generalization and discrimination. J. Exp. Psychol. Anim. Behav. Processes 1: 3-21.

Boakes, R.A. and M.S. Halliday. 1972. Inhibition and learning. Academic Press, London, UK.

Bower, J.M. and J. Kassel. 1990. Variability in tactile projection patterns to cerebellar folia Crus IIA in the Norway rat. J. Comp. Neurol. 302: 768-778.

Bullock, D., J. Fiala, and S.A. Grossberg. 1994. A neural model of timed response learning in the cerebellum. Neural Networks 7: 1101-1114.

Buonomano, D.V. and M.D. Mauk. 1994. Neural network model of the cerebellum: Temporal discrimination and the timing of motor responses. Neural Comp. 6: 38-55.

Chan-Palay, V. 1977. Cerebellar dentate nucleus:

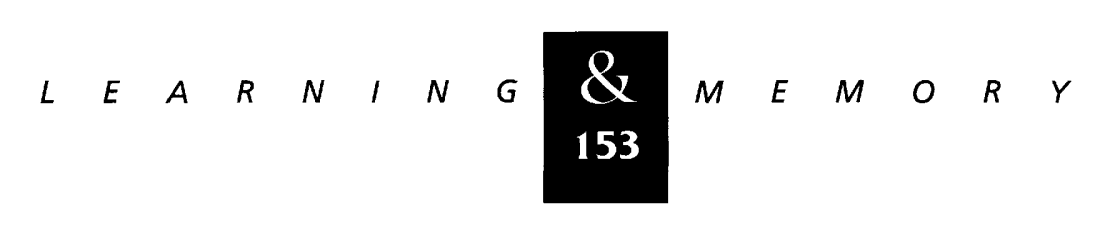




\section{Mauk and Donegan}

Organization, cytology and transmitters. Springer-Verlag, New York, NY.

Chen, C. and R.F. Thompson. 1995. Temporal specificity of long-term depression in parallel-fiber-Purkinje synapses in rat cerebellar slice. Learn. \& Mem. 2: 185-198.

Chen, C., M. Kano, A. Abeliovich, L. Chen, S. Bao, J.J. Kim, K. Hashimoto, R.F. Thompson, and S. Tonegawa. 1995. Impaired motor coordination correlates with persistent multiple climbing fiber innervation in PKC $\gamma$ mutant mice. Cell 83: 1233-1242.

Chen, L., B. Shaowen, L.M. Lockard, J.J. Kim, and R.F. Thompson. 1996. Impaired classical eyeblink conditioning in cerebellar-lesioneu and Purkinje cell degeneration (pcd) mutant mice. J. Neurosci. 16: 2829-2838.

Coleman, S.R. and I. Gormezano. 1971. Classical conditioning of the rabbit's (Oryctolagus cuniculus) nictitating membrane response under symmetrical CS-US interval shifts. J. Comp. Physiol. Psychol. 77: 447-455.

de Zeeuw, C.I., J.C. Holstege, T.J. Ruigrok, and J. Voogd. 1989. Ultrastructural study of the GABAergic, cerebellar, and mesodiencephalic innervation of the cat medial accessory olive: Anterograde tracing combined with immunocytochemistry. J. Comp. Neurol. 284: 12-35.

Donegan, N.H., M.A. Gluck, and R.F. Thompson. 1989. Integrating behavioral and biological models of classical conditioning. In Computational models of learning in simple neural systems (ed. R.D. Hawkins and G.H. Bower), pp. 109-156. Academic Press, New York, NY.

Dudek, S.M. and M.F. Bear. 1993. Bidirectional long-term modification of synaptic effectiveness in the adult and immature hippocampus. J. Neurosci. 13: 2910-2918.

Eccles, J.C., M. Ito, and J. Szentágothai. 1967. The cerebellum as a neuronal machine. Springer-Verlag, New York, NY.

Frey, P.W. and L.E. Ross. 1968. Classical conditioning of the rabbit eyelid response as a function of interstimulus interval. J. Comp. Physiol. Psychol. 65: 246-250.

Gao, J.-H., L.M. Parsons, J.M. Bower, J. Xiong, J. Li, and P.T. Fox. 1996. Cerebellum implicated in sensory acquisition and discrimination rather than motor control. Science 272: 545-547.

Gluck, M.A., E.S. Reifsnider, and R.F. Thompson. 1990. Adaptive signal processing and the cerebellum: Models of classical conditioning and VOR adaptation. In Neuroscience and connectionist theory (ed. M.A. Gluck and D.E. Rumelhart), pp. 131-186. Lawrence Earlbaum Associates, Hillsdale, NJ.

Godlett, C.R., K.M. Hamre, and J.R. West. 1992. Dissociation of spatial navigation and visual guidance performance in Purkinje cell degeneration (pcd) mutant mice. Behav. Brain Res. 47: 129-141.
Goldowitz, D. and L.M. Eisenman. 1992. Genetic mutations affecting murine cerebellar structure and function. In Genetically defined animal models of neurobehavioral dysfunctions (ed. P. Driscoll), pp. 66-88. Birkauser, Boston, MA.

Gormezano, I. 1972. Investigations of defense and reward conditioning in the rabbit. In Classical conditioning II: Current theory and research (ed. A.H. Black and W.F. Prokasy), pp. 151-181. Appleton-Century Crofts, New York, NY.

Gormezano, I., E.J. Kehoe, and B.S. Marshall. 1983. Twenty years of classical conditioning with the rabbit. Prog. Psychobiol. Physiol. Psychol. 10: 197-275.

Gormezano, I., W.F. Prokasy, and R.F. Thompson. 1987. Classical conditioning, 3rd ed. Lawrence Earlbaum Associates, Hillsdale, NJ.

Groenewegen, H.J. and J. Voogd. 1977. The parasagittal zonation within the olivocerebellar projection. I. Climbing fiber distribution in the vermis of cat cerebellum. J. Comp. Neurol. 174: 417-488.

Groenewegen, H.J., J. Voogd, and S.L. Freedman. 1979. The parasagittal zonation within the olivocerebellar projection. II. Climbing fiber distribution in the intermediate and hemispheric parts of cat cerebellum. J. Comp. Neurol. 183: $551-602$.

Grossberg, S. and N.A. Schmajuk. 1989. Neural dynamics of adaptive timing and temporal discrimination during associative learning. Neural Networks 2: 79-102.

Hawkins, R.D. and E.R. Kandel. 1984. Is there a cell-biological alphabet for simple forms of learning? $P_{s y c h}$. Rev. 91: 375-391.

Houk, J.C. and A.R. Gibson. 1986. Sensorimotor processing through the cerebellum. In New concepts in cerebellar neurobiology (ed. J.S. King and J. Courville), pp. 387-416. Lissman, New York, NY.

Hull, C.L. 1943. Principles of behavior. Appleton-CenturyCrofts, New York, NY.

Ito, M. 1982. Cerebellar control of the vestibulo-ocular reflex-around the flocculus hypothesis. Annu. Rev. Neurosci. 12: 85-102.

1984. The cerebellum and neural control. Raven Press, New York, NY.

1988. Long term depression. Annu. Rev. Neurosci. 12: $85-102$.

Ito, M. and M. Kano. 1982. Long lasting depression of parallel fiber-Purkinje cell transmission induced by conjunctive stimulation of parallel fibers and climbing fibers in the cerebellar cortex. Neurosci. Lett. 33: 253-258.

Ito, M., M. Sakurai, and P. Tongroach. 1982. Climbing fibre

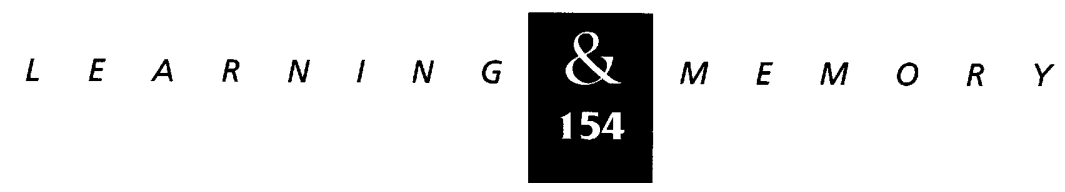


induced depression of both mossy fibre responsiveness and glutamate sensitivity of cerebellar Purkinje cells. J. Physiol. 324: $113-134$.

Jarrard, L.E. and T.L. Davidson. 1991. On the hippocampus and learned conditional responding: effects of aspiration versus ibotenate lesions. Hippocampus 1: 107-117.

Kamin, L.J. 1969. Selective association and conditioning. In Fundamental issues in associative learning (ed. N.H. Mackintosh and W.H. Honig) pp. 276-296, Dalhousie, Halifax, Nova Scotia, Canada.

Kano, M. and M. Kato. 1988. Mode of induction of long-term depression at parallel fibre-Pukinje synapses in the rabbit cerebellar cortex. Neurosci. Res. 5: 544-556.

Keating, J.G. and W.T. Thach. 1995. Nonclock behavior of inferior olive neurons: Interspike interval of Purkinje cell complex spike discharge in the awake behaving monkey is random. J. Neurophysiol. 73: 1329-1340.

Kehoe, E.J. 1988. A layered network model of associative learning: Learning to learn and configuration. Psychol. Rev. 95: $411-433$.

Kehoe, E.J. and R.M. Napier. 1991a. In the blink of an eye: Real time stimulus factors in delay and trace classical conditioning of the rabbit's nictitating membrane response. Quart. J. Exp. Psych. 43B: 257-277.

1991b. Real time factors in the rabbit's nictitating membrane response to pulsed and serial conditioned stimuli. Anim. Learn. Behav. 14: 144-154.

Kehoe, E.J., P. Graham-Clarke, and B.G. Schreurs. 1989. Temporal patterns of the rabbit's nictitating membrane response to compound and component stimuli under mixed CS-US intervals. Behav. Neurosci. 103: 283-295.

Kehoe, E.J., P.S. Horne, and A.J. Horne. 1993a. Discrimination learning using different CS-US intervals in classical conditioning of the rabbit's nictitating membrane response. Psychobiol. 21: 277-285.

Kehoe, E.J., P.S. Horne, M. Macrae, and A.J. Horne. 1993b. Real-time processing of serial stimuli in classical conditioning of the rabbit's nictitating membrane response. J. Exp. Psychol. Anim. Behav. Processes 19: 265-283.

Kelly, T.M., C.C. Zuo, and R.J. Bloedel. 1990. Classical conditioning of the eyeblink reflex in the decerebrate-decerebellate rabbit. Behav. Brain Res. 38: 7-18.

Krupa, D.J., J.K. Thompson, and R.F. Thompson. 1993. Localization of a memory trace in the mammalian brain. Science 260: 989-991.

Lavond D.G. and J.E. Steinmetz. 1989. Acquisition of classical conditioning without cerebellar cortex. Behav. Brain Res. 33: 113-164.

Lavond D.G., J.E. Steinmetz, and M.H. Yokaitis. 1987.
Reacquisition of classical conditioning after removal of cerebellar cortex. Exp. Brain Res. 67: 569-593.

Lavond, D.G, J.J. Kim, and R.F. Thompson. 1993.

Mammalian substrates of aversive classical conditioning. Annu. Rev. Psychol. 44: 317-342.

Linden, D.J. and J.A. Connor. 1991. Participation of postsynaptic PKC in cerebellar long-term depression in culture. Science 254: 1656-1659.

Linden, D.J., M.H. Dickenson, M. Smeyne, and J.A. Connor. 1991. A long-term depression of AMPA currents in cultured cerebellar Purkinje neurons. Neuron 7: 81-89.

Lisberger, S.G. 1988. The neural basis for learning simple motor skills. Science 242: 728-735.

Llinás, R. and M. Mühlethaler. 1988. Electrophysiology of the Guinea-pig cerebellar nuclear cells in the in vitro brainstem-cerebellar preparation. J. Physiol. 404: 241-258.

Llinás, R. and J.P. Welsh. 1993. On the cerebellum and motor learning. Curr. Opin. Neurobiol. 3: 958-968.

Lorenz, E. 1963. Deterministic nonperiodic flow. J. Atmospheric Sci. 20: 130-141.

Lubow, R.E. 1973. Latent inhibition. Psychol. Bull. 79: 398-407.

Mackintosh, N.J. 1975. A theory of attention: Variations in the associability of stimuli with reinforcement. Psychol. Rev. 82: $276-298$.

Mamounas, L.A., R.F. Thompson, and J.I. Madden. 1987. Cerebellar GABAergic processes: Evidence for critical involvement in a form of simple associative learning in the rabbit. Proc. Natl. Acad. Sci. 84: 2101-2105.

Marr, D. 1969. A theory of cerebellar cortex. J. Physiol. 202: $437-470$.

Mauk, M.D. 1997. Relative contributions of cerebellar cortex and nuclei to motor learning: Contradictions or clues? Neuron 18: 343-346.

Mauk, M.D. and R.F. Thompson. 1987. Retention of classically conditioned eyelid responses following acute decerebration. Brain Res. 403: 89-95.

Mauk, M.D. and B.P. Ruiz. 1992. The learning-dependent timing of Pavlovian eyelid responses: Differential conditioning using multiple inter-stimulus intervals. Behav. Neurosci. 106: 666-681.

Mauk, M.D., J.E. Steinmetz, and R.F. Thompson. 1986. Classical conditioning using stimulation of the inferior olive as the unconditioned stimulus. Proc. Natl. Acad. Sci. 83: 5349-5353.

McCormick, D.A. and R.F. Thompson. 1984. Cerebellum:

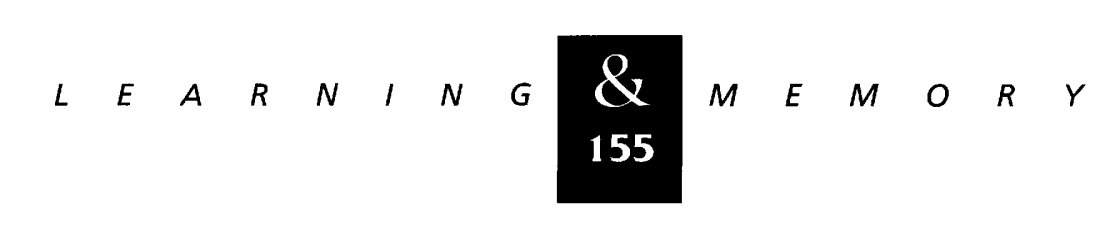




\section{Mauk and Donegan}

Essential involvement in the classically conditioned eyelid response. Science 223: 296-299.

McCormick, D.A., J.E. Steinmetz, and R.F. Thompson. 1985. Lesions of the inferior olivary complex cause extinction of the classically conditioned eyeblink response. Brain Res. 359: $120-130$.

Miles, F.A. and S.G. Lisberger. 1981. Plasticity in the vestibulo-ocular reflex: A new hypothesis. Annu. Rev. Neurosci. 4: 273-299.

Millenson, J.R., E.J. Kehoe, and I. Gormezano. 1977. Classical conditioning of the rabbit's nictitating membrane response under fixed and mixed CS-US intervals. Learn. \& Motiv. 8: 351-366.

Montarolo, P.G., M. Palestini, and P. Strata. 1982. The inhibitory effect of the olivocerebellar input on the cerebellar Purkinje cells in the rat. J. Physiol. 332: 187-202.

Moore, J.W. 1972. Stimulus control: Studies of auditory generalization in rabbits. In Classical conditioning II: Current theory and research (ed. A.H. Black and W.F. Prokasy), pp. 206-230. Appleton-Century Crofts, New York, NY.

Moore, J.W. and D.E.J. Blazis. 1989. Simulation of a classically conditioned response: A cerebellar neural network implementation of the Sutton-Barto-Desmond model. In Neural models of plasticity: Experimental and theoretical approaches (ed. J.H. Byrne and W.O. Barry), pp. 187-207. Academic Press, New York, NY.

Moore, J.W., J.E. Desmond, N.E. Berthier, R.S. Sutton, and A.G. Barto. 1986. Simulation of the classically conditioned nictitating membrane response by a neuron-like adaptive element: Response topography, neuronal firing, and interstimulus intervals. Behav. Brain Res. 21: 143-154.

Moore, J.W., J.E. Desmond, and N.E. Berthier. 1989. Adaptively timed conditioned responses and the cerebellum: A neural network approach. Biol. Cybern. 62: 17-28.

Moyer, J.R., Jr., R.A. Deyo, and J.F. Disterhoft. 1990 Hippocampectomy disrupts trace eye-blink conditioning in rabbits. Behav. Neurosci. 104: 243-252.

Mulkey, R.M. and R.C. Malenka. 1992. Mechanisms underlying induction of homosynaptic long-term depression in area CA1 of the hippocampus. Neuron 9: 967-975.

Napier, R.M., M. Macrae, and E.J. Kehoe. 1992. Rapid reacquisition in conditioning of the rabbit's nictitating membrane response. J. Exp. Psych. Anim. Behav. Processes 18: $182-192$.

Nordholm, A.F., B.J. Thompson, C. Dersarkissian, and R.F. Thompson. 1993. Lidocaine infusion in a critical region of cerebellum completely prevents learning of the conditioned eyeblink response. Behav. Neurosci. 107: 882-886.

Oscarsson, O. 1980. Functional organization of olivary projection to the cerebellar anterior lobe. in The inferior olivary nucleus anatomy and physiology (ed. J. Courville, C. de Montigny, and Y. Lamarre), pp. 279-289. Raven Press, New York, NY.

Palay, S.L. 1974. Cerebellar cortex: Cytology and organization Springer, New York, NY.

Pavlov, I.P. 1927. Conditioned reflexes (translated by G. V. Anrep). Oxford University Press, London, UK.

Pearce, J.M. and G. Hall. 1980. A model for Pavlovian learning: Variations in the effectiveness of conditioned but not unconditioned stimuli. Psychol. Rev. 87: 532-552.

Pellegrini, J.J. and C. Evinger. 1995. The trigeminally evoked blink reflex. 11. Mechanisms of paired-stimulus suppression. Exp. Brain Res. 107: 181-196.

Perrett, S.P. and M.D. Mauk. 1995. Extinction of conditioned eyelid responses requires anterior lobe of cerebellar cortex. J. Neurosci. 15: 2074-2080.

Perrett, S.P., B.P. Ruiz, and M.D. Mauk. 1993. Cerebellar cortex lesions disrupt the learning-dependent timing of conditioned eyelid responses. J. Neurosci. 13: 1708-1718.

Racine R.J., D.A. Wilson, R. Gingell, and D. Sunderland. 1986. Long-term potentiation in the interpositus and vestibular nuclei in the rat. Exp. Brain Res. 63: 158-162.

Raymond, J.L., S.G. Lisberger, and M.D. Mauk. 1996. The cerebellum: A neuronal learning machine? Science 272: 1126-1131.

Rescorla, R.A. 1976. Stimulus generalization: Some predictions from a model of Pavlovian conditioning. J. Exp. Psychol. Anim. Behav. Processes 2: 88-96.

Rescorla, R.A. and A.R. Wagner. 1972. A theory of Pavlovian conditioning: Variations in the effectiveness of reinforcement and nonreinforcement. In Classical conditioning II: Current theory and research (ed. A.H. Black and W.F. Prokasy), Appleton-Century-Crofts, New York, NY.

Robinson, D.A. 1976. Adaptive gain control of the vestibulo-ocular reflex by the cerebellum. J. Neurophysiol. 39: 954-969.

Rudy, J.W. and R.J. Sutherland. 1995. Configural association theory and the hippocampal formation: An appraisal and reconfiguration. Hippocampus 5: 375-389.

Ruigrok, T.J. and J. Voogd. 1990. Cerebellar nucleo-olivary projections in the rat: An anterograde tracing study with Phaseolus vulgaris-leucoagglutinin (PHA-L). J. Comp. Neurol. 298: 315-333.

Sakurai, M. 1987. Synaptic modification of parallel fibre-Purkinje cell transmission in vitro guinea-pig cerebellar slices. J. Physiol. (Lond.) 394: 463-480.

Salafia, W.R., L.J. Martino, K. Cloutman, and A.G. Romano. 1979. Unconditioned-stimulus locus and 
interstimulus-interval shift in rabbit (Oryctolagus cuniculus) nictitating membrane conditioning. Pavlovian J. Biol. Sci. 14: $64-71$.

Salin, P., R.C. Malenka, and R.A. Nicoll. 1996. Cyclic AMP mediates a presynaptic form of LTP at cerebellar parallel fiber synapses. Neuron 16: 797-803.

Schmajuk, N.A. and J.J. DiCarlo. 1992. Stimulus configuration, classical conditioning, and hippocampal function. Psychol. Rev. 99: 268-305.

Schneiderman, N. 1966. Interstimulus interval function of the nictitating membrane response in the rabbit under delay versus trace conditioning. J. Comp. Physiol. Psychol. 62: 397-402.

Schneiderman, N. and I. Gormezano. 1964. Conditioning of the nictitating membrane of the rabbit as a function of CS-US interval. J. Comp. Physiol. Psychol. 57: 188-195.

Schreurs, B.G. and D.L. Alkon. 1993. Rabbit cerebellar slice analysis of long-term depression and its role in classical conditioning. Brain Res. 631: 235-240.

1996. Pairing-specific long-term depression of Purkinje cell excitatory postsynaptic potentials results from a classical conditioning procedure in the rabbit cerebellar slice. J. Neurophysiol. 75: 1051-1060.

Shibuki, K. and D. Okada. 1992. Cerebellar long-term potentiation under suppressed postsynaptic $\mathrm{Ca}^{2+}$ activity. NeuroReport 3: 231-234.

Siegel. S. 1972. Latent inhibition in eyelid conditioning. In Classical conditioning II: Current theory and research (ed. A.H. Black and W.F Prokasy), pp. 231-247.

Appleton-Century-Crofts, New York, NY.

Smith, M.C. 1968. CS-US interval and US intensity in classical conditioning of the rabbit's nictitating membrane response. J. Comp. Physiol. Psychol. 66: 679-687.

Smith, M.C., S.R. Coleman, and I. Gormezano. 1969. Classical conditioning of the rabbit's nictitating membrane response at backward, simultaneous and forward CS-US intervals. J. Comp. Physiol. Psychol. 69: 226-231.

Solomon, P.R. 1977. Tole of the hippocampus in blocking and conditioned inhibition of the rabbit's nicitating membrane response. J. Comp. Physiol. Psychol. 91: 407-417.

Solomon, P.R. and J.W. Moore. 1975. Latent inhibition and stimulus generalization of the classically conditioned nictitating membrane response in rabbits (Oryctolagus cuniculus) following dorsal hippocampal lesions. J. Comp. Physiol. Psych. 89: 1192-1203.

Steinmetz, J.E. 1990. Classical nictitating membrane conditioning in rabbits with varying interstimulus intervals and direct activation of cerebellar mossy fibers as the CS. Behav. Brain Res. 38: 97-108.
Steinmetz, J.E., C.G. Logan, D.J. Rosen, B.J. Thompson, D.G. Lavond, and R.F. Thompson. 1987. Initial localization of the acoustic conditioned stimulus projection system to the cerebellum during classical eyelid conditioning. Proc. Natl. Acad. Sci. 84: 3531-3535.

Steinmetz, J.E., C.G. Logan, and R.F. Thompson. 1988. Essential involvement of mossy fibers in projecting the CS to the cerebellum during classical conditioning. In Cellular mechanisms of conditioning and behavioral plasticity (ed. C.D. Woody, D.L. Alkon, and J.L. McGaugh), Plenum Press, New York, NY.

Steinmetz, J.E., D.G. Lavond, and R.F. Thompson. 1989. Classical conditioning in rabbits using pontine nucleus stimulation as a conditioned stimulus and inferior olive stimulation as an unconditioned stimulus. Synapse 3: $225-233$

Sutherland, R.J., R.J. McDonald, C.R. Hill, and J.W. Rudy. 1989. Damage to the hippocampal formation in rats selectively impairs the ability to learn cue relationships. Behav. Neural Biol. 52: 331-356.

Sutton, R.S. and A.G. Barto. 1981. Toward a modern theory of adaptive networks: Implications for serial and second order conditioning. J. Exp. Psychol. Anim. Behav. Processes 5: 43-64.

1990. Time-derivative models of Pavlovian reinforcement. In Learning and computational neuroscience (ed. M. Gabriel and J.W. Moore), pp. 497-537. MIT Press, Cambridge, MA.

Thach, W.T. 1980. Complex spikes, the inferior olive, and natural behavior. In The inferior olivary nucleus: Anatomy and physiology (ed. J. Courville, C. de Montigny, and Y. Lamarre), pp. 349-360. Raven Press, New York, NY.

Thach, W.T., J.P. Goodkin, and J.G. Keating. 1992.

Cerebellum and the adaptive coordination of movement. Annu. Rev. Neurosci. 15: 403-442.

Thompson, R.F. 1986. The neurobiology of learning and memory. Science 233: 941-947.

Thompson, R.F. and D.J. Krupa. 1994. Organization of memory traces in the mamalian brain. Annu. Rev. Neurosci. 17: 519-549.

Triarhou, L.C. and B. Ghetti. 1991. Stabilization of neurone number in the inferior olive complex of aged "Purkinje cell degeneration" mutant mice. Acta Neuropathol. 81: 597-602.

Wagner, A.R. 1969. Stimulus selection and a "modified continuity theory." In The psychology of learning and motivation (ed. G.H. Bower and J.T. Spence), pp. 1-43. Academic Press, New York, NY.

1976. Priming in STM: An information-processing mechanism for self-generated or retrieval-generated depression of performance. In Habituation: Perspectives from child development, animal behavior, and neurophysiology

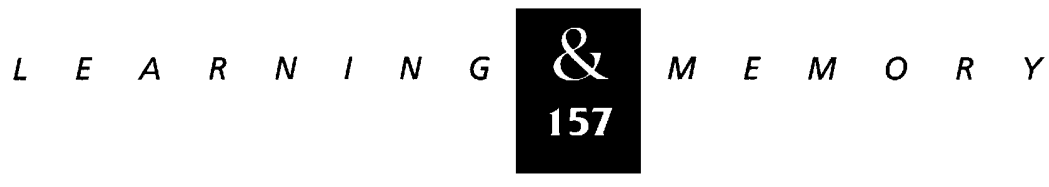


Mauk and Donegan

(ed. T.J. Tighe and R.N. Leaton), pp. 95-128. Lawrence Earlbaum Associates, Hillsdale, NJ.

1979. Habituation and memory. In Mechanisms of learning and motivation: A memorial to Jerzy Konorski (ed. A. Dickinson and R.A. Boakes), pp. 53-82. Lawrence Earlbaum Associates, Hillsdale, NJ.

1981. SOP: A model of automatic memory processing in animal behavior. In Information processing in animals (ed. N.E. Spear and R.R. Miller), pp. 5-47. Lawrence Earlbaum Asociates, Hillsdale, NJ.

Wagner, A.R. and R.A. Rescorla. 1972. Inhibition in Pavlovian conditioning: Application of a theory. In Inhibition and learning (ed. R.A. Boakes and M.S. Halliday), pp. 301-336. Academic Press, London, UK.

Welsh, J.P. and J.A. Harvey. 1989. Cerebellar lesions and the nictitating membrane reflex: Performance deficits of the conditioned and unconditioned response. J. Neurosci. 9: $299-311$.

1991. Pavlovian conditioning in the rabbit during inactivation of the interpositus nucleus. J. Physiol. 444: $459-480$.

1992. The role of the cerebellum in voluntary and reflexive movements: History and current status. In The cerebellum revisited (ed. R. Llinas and C. Sotelo), pp. 301-334. Springer-Verlag, New York, NY.

Whitlow, J.W. and A.R. Wagner. 1972. Negative patterning in classical conditioning: Summation of response tendencies to isolable and configural components. Psychon. Sci. 27: 299-301.

Yeo, C.H. and M.J. Hardiman. 1992. Cerebellar cortex and eyeblink conditioning: A reexamination. Exp. Brain Res. $8: 623-638$.

Yeo, C.H., M.J. Hardiman, and M. Glickstein. 1985a. Classical conditioning of the nictitating membrane response of the rabbit. I. Lesions of the cerebellar nuclei. Exp. Brain Res. 60: 87-98.

1985b. Classical conditioning of the nictitating membrane response of the rabbit. II. Lesions of the cerebellar cortex. Exp. Brain Res. 60: 99-113.

1986. Classical conditioning of the nictitating membrane response of the rabbit. IV. Lesions of the inferior olive. Exp. Brain Res. 63: 81-92.

Received March 11, 1997; accepted in revised form April 30, 1997.

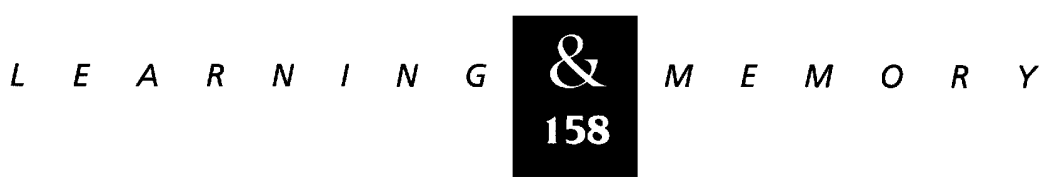




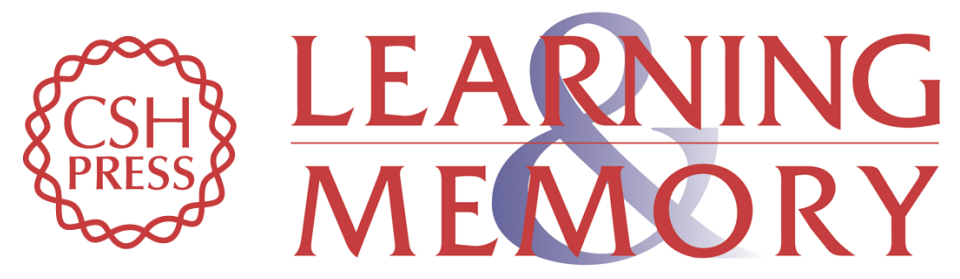

\section{A model of Pavlovian eyelid conditioning based on the synaptic organization of the cerebellum.}

M D Mauk and N H Donegan

Learn. Mem. 1997, 4:

Access the most recent version at doi:10.1101//m.4.1.130

References This article cites 106 articles, 16 of which can be accessed free at:

http://learnmem.cshlp.org/content/4/1/130.full.html\#ref-list-1

License

Email Alerting Receive free email alerts when new articles cite this article - sign up in the box at the Service top right corner of the article or click here. 